\title{
Analysis and Applications of a Generalized Finite Element Method with Global-Local Enrichment Functions ${ }^{\star}$
}

\author{
C.A. Duarte ${ }^{1}$ and D.-J. Kim \\ Department of Civil and Environmental Engineering, University of Illinois at Urbana-Champaign \\ 2122 Newmark Laboratory, 205 North Mathews Avenue \\ Urbana, Illinois 61801, USA
}

\section{SUMMARY}

This paper presents a procedure to build enrichment functions for partition of unity methods like the generalized finite element method and the $h p$ cloud method. The procedure combines classical globallocal finite element method concepts with the partition of unity approach. It involves the solution of local boundary value problems using boundary conditions from a global problem defined on a coarse discretization. The local solutions are in turn used to enrich the global space using the partition of unity framework. The computations at local problems can be parallelized without difficulty allowing the solution of large problems very efficiently.

The effectiveness of the approach in terms of convergence rates and computational cost is investigated in this paper. We also analyze the effect of inexact boundary conditions applied to local problems and the size of the local domains on the accuracy of the enriched global solution.

Key aspects of the computational implementation, in particular, the numerical integration of generalized FEM approximations built with global-local enrichment functions, are presented.

The method is applied to fracture mechanics problems with multiple cracks in the domain. Our numerical experiments show that even on a serial computer the method is very effective and allows the solution of complex problems. Our analysis also demonstrates that the accuracy of a global problem defined on a coarse mesh can be controlled using a fixed number of global degrees of freedom and the proposed global-local enrichment functions.

KEY WORDS: Generalized finite element method, extended finite element method, meshfree, globallocal, fracture mechanics, multiple cracks.

\section{Introduction}

The effectiveness of partition of unity methods like $h p$ clouds [25, 26], generalized [7, 19, 20, $32,40,49,50]$, and extended [10, 34] finite element methods relies, to a great extent, on the proper selection of enrichment functions. Customized enrichment functions can be used to

\footnotetext{
${ }^{1}$ Correspondence to: C.A. Duarte, Department of Civil and Environmental Eng., University of Illinois at Urbana-Champaign, Newmark Laboratory, 205 North Mathews Avenue, Urbana, Illinois 61801, USA. Tel.: +1-217-244-2830; Fax: +1-217-333-3821. e-mail: caduarte@uiuc.edu.

* Submitted to Computer Methods in Applied Mechanics and Engineering (November 30, 2006)
} 
model local features in a domain like cracks [15, 21, 34, 35, 39, 41, 55], edge singularities [20], boundary layers [17], inclusions [54], voids [50, 54], microstructures [33, 47], etc., instead of strongly refined meshes with elements faces/edges fitting the local features, as required in the finite element method. This has lead to a growing interest on this class of methods by the engineering community.

The generalized/extended FEM has the ability to analyze crack surfaces arbitrarily located within the mesh (across finite elements). Figure 1(b) shows the representation of a threedimensional crack surface using the generalized finite element method presented in [23, 45]. The generalized FEM enjoys, for this class of problems, the same level of flexibility and user

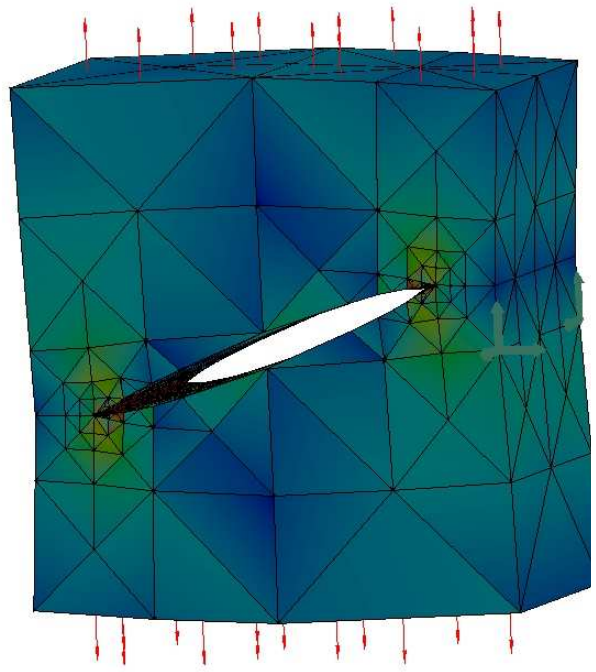

(a)

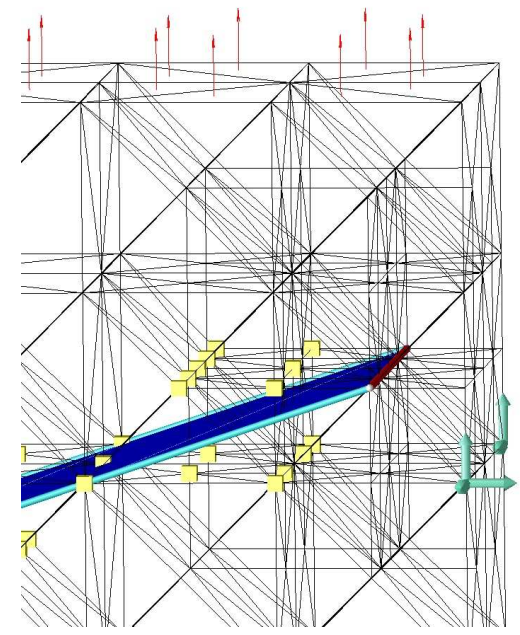

(b)

Figure 1. (a) A three-dimensional GFEM discretization for a body with an inclined cracked. A finer mesh is required near the crack front to compensate the limitations of the enrichment functions. (b) Three-dimensional view of the crack front. Notice that the crack surface goes through the elements.

friendliness as meshfree methods while being more computationally efficient.

In simple two-dimensional cases, a crack can be accurately modeled in partition of unity methods using enrichment functions from the asymptotic expansion of the elasticity solution in the neighborhood of a crack. The three-dimensional case is, however, not as straightforward. The intersection of a crack surface with the boundary of the domain, creates complex stress distributions. Asymptotic forms of the elasticity solution near edges in three dimensional problems are discussed in, for example, $[29,36]$. There are terms in the three dimensional expansions which are very similar to the two-dimensional case. Nevertheless, the theory is quite complex and not practical for engineering applications.

Currently, two dimensional expansions of the elasticity solution are used as enrichment functions for three-dimensional cracks in finite size domains [21, 35, 55]. As a consequence, a sufficiently fine mesh must be used around the crack front to achieve acceptable accuracy. An example is shown in Figure 1(a). Even though the refinement does not have to be as strong as 
in the finite element method, it still creates many of the problems faced by the finite element method when simulating propagating cracks. In particular $(i)$ Increased computational cost by re-solving the problem from scratch after each crack propagation step. Realistic simulations require hundreds of crack propagation steps, several different initial crack configurations, and many times, a non-linear analysis. Problems like multi-site damage analysis [3] would also benefit from a methodology able to avoid re-solutions from scratch after small changes in input data; (ii) Mapping of solutions between meshes when solving non-linear or timedependent problems. Mappings between non-nested meshes is costly and may lead to a loss of accuracy even if fine meshes are used. These limitations offset some of the advantages of generalized/extended FEMs for several classes of problems.

The authors have recently proposed a procedure to build enrichment functions for partition of unity methods and, in particular, for the generalized FEM [18, 22]. The approach presented in $[18,22]$ was geared towards three-dimensional fracture mechanics problems but the methodology can also be applied to many other classes of problems. In this procedure, enrichment functions are computed from the solution of local boundary value problems defined in a neighborhood of a crack front in three-dimensions. The approach uses concepts from the classical global-local finite element method, where boundary conditions for local problems are extracted from the solution of a global problem [37]. However, unlike the classical global-local FEM, the approach presented in $[18,22]$ is able to account for local-global interactions and interactions among local features, like multiple cracks. The approach allows the use of coarse global meshes around crack fronts while delivering accurate solutions and is specially appealing to evolution type problems like propagating cracks. Numerical experiments demonstrating the computational efficiency and accuracy of the method were presented in [18, 22].

In this paper, we present a detailed numerical analysis of the procedure presented in $[18,22]$. In particular, we investigate the following issues

(i) The effect of using the solution of the global problem, instead of the unknown exact solution, as boundary conditions for the local problems (Section 4.1.1);

(ii) The effect of the size of the local domains on the performance of the proposed GFEM with global-local enrichment functions. Larger domains are in principle desirable since they would reduce the effect of not using exact boundary conditions for the local problems. However, large domains are more computationally demanding (Section 4.1.2);

(iii) The effectiveness of the proposed technique in terms of convergence rates of the global problem and its relations with the convergence rates of the local problems (Section 4.2);

(iv) The computational cost of the procedure as compared with existing methodologies (Section 4.3).

In addition, we also discuss some key aspects of the computational implementation, in particular, the numerical integration of generalized FEM approximations built with globallocal enrichment functions (Cf. Section I.3). The main conclusions of this work are presented in Section 5.

\section{The Generalized Finite Element Method}

In this section, we briefly review the construction of the so-called generalized finite element approximations. Further details can be found in, for example, [6, 7, 20, 40, 51].

[global-local'GFEM`duarte 'kim`R1 - August 18, 2007] 
The generalized FEM $[6,7,20,40,51]$ is an instance of the so-called partition of unity method. This method has its origins in the works of Babuška et al. [5, 7, 32] (under the names "special finite element methods", "generalized finite element method" and "partition of unity finite element method") and Duarte and Oden [16, 24, 25, 26, 40] (under the names "hp clouds" and "cloud-based $h p$ finite element method"). The extended FEM [10,34] and several of the so-called meshfree methods proposed in recent years can also be viewed as special cases of the partition of unity method. The shape functions, $\phi_{\alpha i}$, in this class of methods are built from the product of a partition of unity, $\varphi_{\alpha}$, and enrichment functions, $L_{\alpha i}$

$$
\phi_{\alpha i}:=\varphi_{\alpha} L_{\alpha i} \quad i \in \mathcal{I}(\alpha) \quad(\text { no sum on } \alpha)
$$

where $\varphi_{\alpha}, \alpha=1, \ldots, N, N$ being the number of functions, constitute a partition of unity, i.e., a set of functions defined in a domain $\Omega$ with the property that $\sum_{\alpha=1}^{N} \varphi_{\alpha}(\boldsymbol{x})=1$ for all $\boldsymbol{x}$ in $\Omega$. The index set of the enrichment functions at a vertex node $\boldsymbol{x}_{\alpha}$ is denoted by $\mathcal{I}(\alpha)$. The support of $\varphi_{\alpha},\left\{\boldsymbol{x}: \varphi_{\alpha}(\boldsymbol{x}) \neq 0\right\}$, is denoted by $\omega_{\alpha}$. In the generalized finite element method, the partition of unity is, in general, provided by linear Lagrangian finite element shape functions. The support $\omega_{\alpha}$ of $\varphi_{\alpha}$ is then given by the union of the finite elements sharing a vertex node $\boldsymbol{x}_{\alpha}$. The resulting shape functions are called generalized finite element shape functions. Figure 2 illustrates the construction of GFEM shape functions. The generalized FEM is equivalent to the standard FEM when polynomial enrichment functions, $L_{\alpha i}$, are used. The main strength of the generalized FEM is its ability of using non-polynomial enrichment functions as illustrated in Figure 2(b). In particular, custom-built enrichment functions that are solutions of local boundary value problems can be used as well. These so-called global-local enrichment functions are described in detail in Section 3.

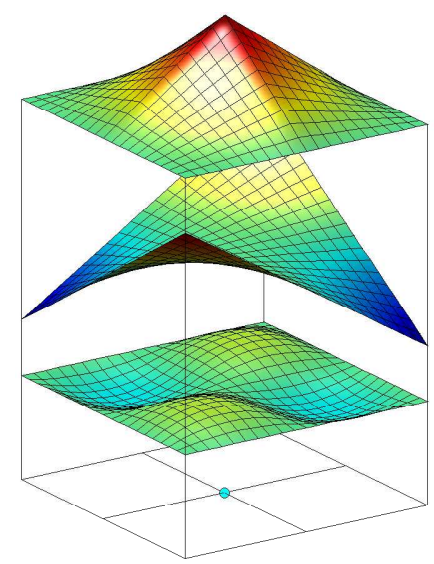

(a)

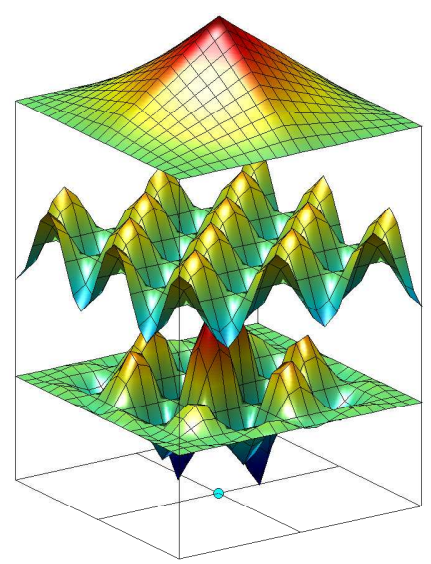

(b)

Figure 2. Construction of a generalized FEM shape function using a polynomial (a) and a nonpolynomial enrichment (b). Here, $\varphi_{\alpha}$ are the functions at the top, the enrichment functions, $L_{\alpha i}$, are the functions in the middle, and the generalized FE shape functions, $\phi_{\alpha i}$, are the resulting bottom functions. 
Local Approximations A partition of unity-based approximation of a scalar field $u(\boldsymbol{x})$ defined on a domain $\Omega \subset \mathbb{R}^{n}, n=1,2,3$, can be written, using shape functions (1), as

$$
u_{h}(\boldsymbol{x})=\sum_{\alpha=1}^{N} \sum_{i \in \mathcal{I}(\alpha)} a_{\alpha i} \phi_{\alpha i}(\boldsymbol{x})=\sum_{\alpha=1}^{N} \varphi_{\alpha}(\boldsymbol{x}) u_{h \alpha}(\boldsymbol{x})
$$

where $a_{\alpha i}, \alpha=1, \ldots, N, i \in \mathcal{I}(\alpha)$, are unknown coefficients and $u_{h \alpha}(\boldsymbol{x}):=\sum_{i \in \mathcal{I}(\alpha)} a_{\alpha i} L_{\alpha i}(\boldsymbol{x})$ denotes a local approximation of the field $u(\boldsymbol{x})$ defined on $\omega_{\alpha}$ and belonging to the local space

$$
\chi_{\alpha}\left(\omega_{\alpha}\right)=\operatorname{span}\left\{L_{\alpha i}(\boldsymbol{x})\right\}_{i \in \mathcal{I}(\alpha)}
$$

where $L_{\alpha i}, i \in \mathcal{I}(\alpha)$, are basis or enrichment functions and $L_{\alpha 1}=1$.

A-Priori Error Estimate An a-priori error estimate for partition of unity approximations and, in particular, for the generalized finite element method, was proved by Babuška and Melenk [7, 32]. The estimate says that if the partition of unity $\operatorname{POU}_{N}=\left\{\varphi_{\alpha}\right\}_{\alpha=1}^{N}$ satisfies some mild requirements and the error of the local approximations, $u_{h \alpha} \in \chi_{\alpha}\left(\omega_{\alpha}\right), \alpha=1, \ldots, N$, are bounded by

$$
\left\|u-u_{h \alpha}\right\|_{E\left(\omega_{\alpha}\right)}<\epsilon(\alpha, u), \quad \alpha=1, \ldots, N,
$$

then the error of a partition of unity approximation, $u_{h}$, defined in (2) is bounded by

$$
\left\|u-u_{h}\right\|_{E(\Omega)}<C\left(\sum_{\alpha=1}^{N} \epsilon^{2}(\alpha, u)\right)^{1 / 2}
$$

where $\|\cdot\|_{E}$ denotes the energy norm and $C$ is a constant. Details and proofs can be found in $[16,26,32]$. If the enrichment functions $L_{\alpha i}, i \in \mathcal{I}(\alpha)$ can (locally) approximate well the function $u$ over $\omega_{\alpha}, \alpha=1, \ldots, N$, the estimate (5) shows that the global approximation $u_{h}$ can approximate well the function $u$ over the (global) domain $\Omega$. The rate of convergence of $u_{h}$ is controlled by the rate of convergence of the local approximations $u_{h \alpha}, \alpha=1, \ldots, N$. In Sections 4.2.1 and 4.2.2 we use this result to analyze the effectiveness of the global-local enrichment functions defined in Section 3.

\section{A Global-Local Approach to Build Enrichment Functions}

In this section, we present a global-local approach to build enrichment functions for the generalized FEM. The approach is based on the global-local formulation presented in [18, 22]. We focus on three-dimensional linear elasticity problems and illustrate the main ideas using fracture mechanics examples. The formulation is, however, applicable to other classes of problems as well. Key aspects of the computational implementation are discussed in Section I.

\subsection{Formulation of Global Problem}

Consider a domain $\bar{\Omega}_{G}=\Omega_{G} \cup \partial \Omega_{G}$ in $\mathbb{R}^{3}$. The boundary is decomposed as $\partial \Omega_{G}=\partial \Omega_{G}^{u} \cup \partial \Omega_{G}^{\sigma}$ with $\partial \Omega_{G}^{u} \cap \partial \Omega_{G}^{\sigma}=\emptyset$. 
The equilibrium and constitutive equations are given by

$$
\nabla \cdot \sigma=0 \quad \sigma=C: \varepsilon \quad \text { in } \Omega_{G},
$$

where $\boldsymbol{C}$ is Hooke's tensor. The following boundary conditions are prescribed on $\partial \Omega_{G}$

$$
\boldsymbol{u}=\overline{\boldsymbol{u}} \text { on } \partial \Omega_{G}^{u} \quad \boldsymbol{\sigma} \cdot \boldsymbol{n}=\overline{\boldsymbol{t}} \text { on } \partial \Omega_{G}^{\sigma},
$$

where $\boldsymbol{n}$ is the outward unit normal vector to $\partial \Omega_{G}^{\sigma}$ and $\overline{\boldsymbol{t}}$ and $\overline{\boldsymbol{u}}$ are prescribed tractions and displacements, respectively.

Let $\boldsymbol{u}_{G}^{0}$ denote a generalized FEM approximation of the solution $\boldsymbol{u}$ of problem (6), (7). The approximation $\boldsymbol{u}_{G}^{0}$ is the solution of the following problem:

Find $\boldsymbol{u}_{G}^{0} \in \boldsymbol{X}_{G}^{h p}\left(\Omega_{G}\right) \subset H^{1}\left(\Omega_{G}\right)$ such that, $\forall \boldsymbol{v}_{G}^{0} \in \boldsymbol{X}_{G}^{h p}\left(\Omega_{G}\right)$

$$
\int_{\Omega_{G}} \boldsymbol{\sigma}\left(\boldsymbol{u}_{G}^{0}\right): \boldsymbol{\varepsilon}\left(\boldsymbol{v}_{G}^{0}\right) d \boldsymbol{x}+\eta \int_{\partial \Omega_{G}^{u}} \boldsymbol{u}_{G}^{0} \cdot \boldsymbol{v}_{G}^{0} d \boldsymbol{s}=\int_{\partial \Omega_{G}^{\sigma}} \overline{\boldsymbol{t}} \cdot \boldsymbol{v}_{G}^{0} d \boldsymbol{s}+\eta \int_{\partial \Omega_{G}^{u}} \overline{\boldsymbol{u}} \cdot \boldsymbol{v}_{G}^{0} d \boldsymbol{s}
$$

where, $\boldsymbol{X}_{G}^{h p}\left(\Omega_{G}\right)$ is a discretization of $H^{1}\left(\Omega_{G}\right)$, the Hilbert space defined on $\Omega_{G}$, built with generalized FEM shape functions and $\eta$ is a penalty parameter. In the computations presented in Section 4 , we adopt $\eta=10^{8} * E * J$, where $E$ and $J$ are the Young's modulus of the material and the Jacobian of a volume element with a face on $\Omega_{G}^{u}$, respectively. We use the penalty method due to its simplicity and generality. Detailed discussion and analysis of methods for the imposition of Dirichlet boundary conditions in generalized FEMs can be found in, e.g., [4]. Problem (8) leads to a system of linear equations for the unknown degrees of freedom of $\boldsymbol{u}_{G}^{0}$. The mesh used to solve problem (8) is typically a coarse quasi-uniform mesh.

\subsection{Local Problems}

Let $\Omega_{l o c}$ denote a subdomain of $\Omega_{G}$. This local domain may contain cracks, holes, inclusions, fibers, or other local feature of interest. In this paper, we focus on the case of local domains containing a single crack surface $\Gamma_{c}$ with front $\Gamma_{\text {front }}$. Details on discretizations used on $\Omega_{l o c}$ are given in Section I.

The following local problem is solved on $\Omega_{l o c}$ after the global solution $\boldsymbol{u}_{G}^{0}$ is computed as described above:

Find $\boldsymbol{u}_{l o c} \in \boldsymbol{X}_{l o c}^{h p}\left(\Omega_{l o c}\right) \subset H^{1}\left(\Omega_{l o c}\right)$ such that, $\forall \boldsymbol{v}_{l o c} \in \boldsymbol{X}_{l o c}^{h p}\left(\Omega_{l o c}\right)$

$$
\begin{aligned}
& \int_{\Omega_{l o c}} \boldsymbol{\sigma}\left(\boldsymbol{u}_{l o c}\right): \boldsymbol{\varepsilon}\left(\boldsymbol{v}_{l o c}\right) d \boldsymbol{x}+\eta \int_{\partial \Omega_{l o c} \backslash\left(\partial \Omega_{l o c} \cap \partial \Omega_{G}^{\sigma}\right)} \boldsymbol{u}_{l o c} \cdot \boldsymbol{v}_{l o c} d \boldsymbol{s}= \\
& \eta \int_{\partial \Omega_{l o c} \backslash\left(\partial \Omega_{l o c} \cap \partial \Omega_{G}\right)} \boldsymbol{u}_{G}^{0} \cdot \boldsymbol{v}_{l o c} d \boldsymbol{s}+\eta \int_{\partial \Omega_{l o c} \cap \partial \Omega_{G}^{u}} \overline{\boldsymbol{u}} \cdot \boldsymbol{v}_{l o c} d \boldsymbol{s}+\int_{\partial \Omega_{l o c} \cap \partial \Omega_{G}^{\sigma}} \overline{\boldsymbol{t}} \cdot \boldsymbol{v}_{l o c} d \boldsymbol{s}
\end{aligned}
$$

where, $\boldsymbol{X}_{l o c}^{h p}\left(\Omega_{l o c}\right)$ is a discretization of $H^{1}\left(\Omega_{l o c}\right)$ using GFEM shape functions.

A key aspect of problem (9) is the use of the generalized FEM solution of the (crude) global problem, $\boldsymbol{u}_{G}^{0}$, as boundary condition on $\partial \Omega_{l o c} \backslash\left(\partial \Omega_{l o c} \cap \partial \Omega_{G}\right)$. The present paper focuses on fracture mechanics problems. The solutions are smooth away from crack fronts where it is possible to assume that the crude solution of a global problem, $\boldsymbol{u}_{G}^{0}$, can approximate the exact solution reasonably well. Our numerical experiments presented in Section 4.1 confirm this. Exact boundary conditions are prescribed on portions of $\partial \Omega_{l o c}$ that intersect either $\partial \Omega_{G}^{u}$ or $\partial \Omega_{G}^{\sigma}$.

[global-local’GFEM'duarte'kim`R1 - August 18, 2007] 
The formulation above considers for simplicity the case of a single local problem per crack in the computational domain. It is also conceivable to define several local problems along each crack front with overlapping local domains. In this case, the boundary of a local problem intersects the crack front. The crude global solution, $\boldsymbol{u}_{G}^{0}$, can not approximate the exact solution well at these points. However, each one of these points at the crack front will be inside of one of the local problems since the local domains overlap. Therefore, the solution of the other local problem will be able to approximate well the exact solution at this point even with non-exact boundary conditions because of the ellipticity of the problem under consideration. This allows us to utilize the crude solution of a global problem for the construction of local problems using the crude solution as boundary conditions.

\subsection{Global-Local Enrichment Functions}

The local solution $\boldsymbol{u}_{l o c}$ is potentially an excellent enrichment function for the global problem defined in Section 3.1. We call $\boldsymbol{u}_{l o c}$ a global-local enrichment function since it is computed using the same approach used in the so-called global-local FEM [37]. We use these functions to define the following vector-valued global shape function

$$
\phi_{\alpha}=\varphi_{\alpha} \boldsymbol{u}_{l o c}
$$

where $\varphi_{\alpha}$ denotes a global partition of unity function. This function is used at nodes $\boldsymbol{x}_{\alpha}$ of the global mesh whose support, $\omega_{\alpha}$, is contained in the local domain $\Omega_{l o c}$. The global problem defined in Section 3.1 is then solved again using these global functions. The solution of this enriched global problem is denoted by $\boldsymbol{u}_{G}^{E}$. The procedure is illustrated with the aid of the edge cracked rectangular bar shown in Figure 3(a). It contains a through-the-thickness edge crack, $\Gamma_{c}$, with front $\Gamma_{\text {front }}$. Figure 3(b) illustrates a local domain extracted from the global mesh shown in Figure 3(a). The boundary conditions applied to a local problem are displacements computed by solving the global problem on a coarse mesh. These boundary conditions are also illustrated in Figure 3(b).

Related approaches The generalized FEM described above is related to global-local techniques developed for the classical finite element method in the 70's [37] and broadly used in many practical applications of the FEM. A fundamental difference, however, is that the proposed generalized FEM accounts for possible interactions of local (near crack, for example) and global (structural) behavior. These interactions can be accounted when solving the global problem with global-local enrichment functions. This is in contrast with standard global-local FEM.

The proposed approach for the construction of enrichment functions is also related to the so-called mesh-based handbook approach of Strouboulis et al. [51, 52, 53] for the solution of Laplace's equation in two-dimensional domains with many voids. The fundamental difference between the two approaches is the boundary conditions used for the local problems. Strouboulis et al. $[51,52,53]$ consider the problem of a scalar equation with microscale. Because the exact solution is not smooth and any coarse solution can not approximate it well, it is not possible in this case to use a crude global solution as boundary conditions for the local problems. Hence harmonic polynomials are used to defined a set of Neumann boundary conditions. The local domains are enlarged by a buffer zone to minimize the effect of inexact boundary conditions.

Our approach is also related to upscaling techniques for microscale problems [30]. However, the proposed approach does not lead to non-conforming approximations like in some upscaling 


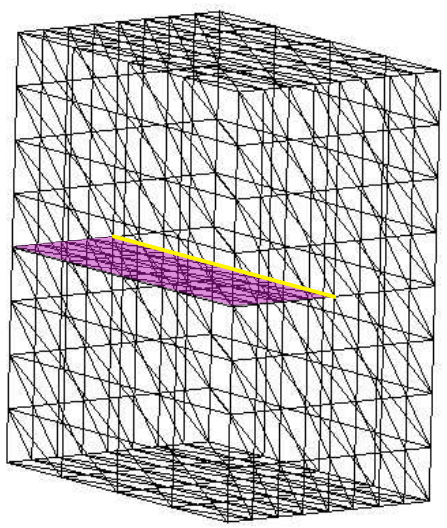

(a) Edge cracked rectangular bar. Mesh for global problem.

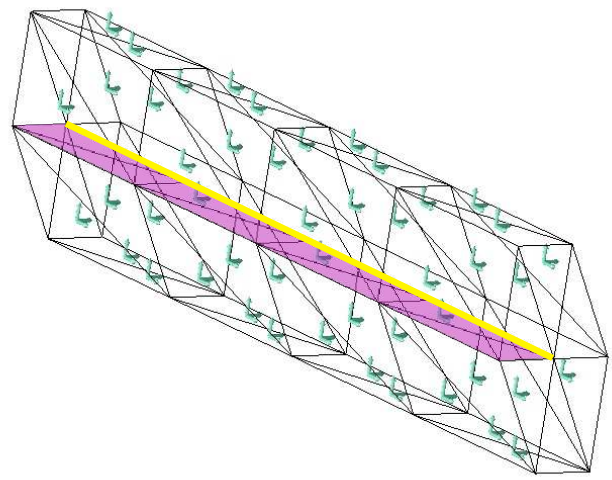

(b) Local domain extracted from the global mesh at the crack front. The vectors on the element faces illustrates the applied displacement boundary conditions.

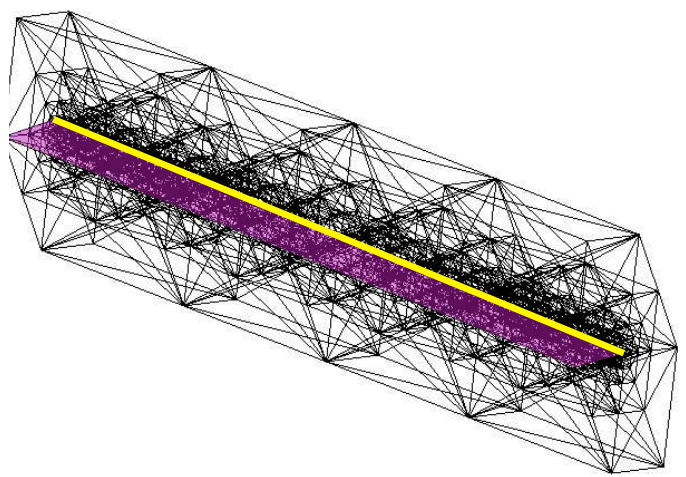

(c) Local mesh refined around the crack front. This mesh is nested in the mesh of the global problem.

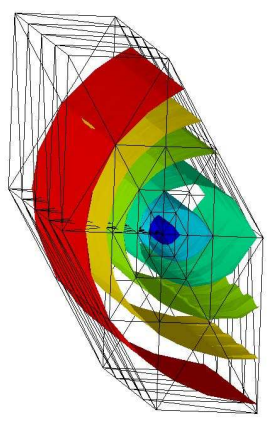

(d) Isosurfaces of the computed solution, $\boldsymbol{u}_{l o c}$, of a local problem.

Figure 3. Construction of global-local enrichment functions for the GFEM. The solution of the local problem shown in (d) is used as an enrichment function for the global problem shown in (a).

techniques [30]. In our case, the global shape functions built with local solution enrichment functions $\boldsymbol{u}_{l o c}$ overlap and are conforming.

\section{Numerical Experiments}

Several numerical experiments are presented in this section with the goal of addressing the following issues in the proposed GFEM with global-local functions:

[global-local`GFEM`duarte`kimR1 - August 18, 2007] 
- The effect of using the solution of the global problem, instead of the unknown exact solution, as boundary conditions for the local problems (Section 4.1.1);

- The effect of the size of the local domains on the performance of the proposed GFEM. Larger domains are desirable in principle since they would reduce the effect of not using exact boundary conditions for local problems. However, large domains are more computationally demanding (Section 4.1.2);

- The effectiveness of the proposed technique in terms of convergence rates of the global problem and their relation to the convergence rates of the local problems (Section 4.2);

- The computational cost of the GFEM with and without global-local enrichment functions (Section 4.3);

- The size of the largest problem that can be solved on a given hardware using the GFEM with and without global-local enrichment functions (Section 4.3).

Discretizations of global and local problems are denoted by

$$
G^{p=\left(p_{x}, p_{y}, p_{z}\right)} \quad \text { and } \quad L_{\text {nref, nlay }}^{p=\left(p_{x}, p_{y}, p_{z}\right)},
$$

respectively, where (i) $p_{x}, p_{y}$ and $p_{z}$ denote the polynomial degree of the shape functions in the $x$-, $y$-, and $z$-direction, respectively; (ii) nlay denotes the number of layers of elements around the crack front in the mesh extracted from the global problem; (iii) nref indicates the level of the local (uniform or non-uniform) mesh refinement. Details on the construction of local discretizations are provided in Section I.1. In all numerical experiments, a single local problem per crack front is defined. All computations were performed on a Dell Dimension 4600 $\mathrm{PC}$ with a $3.2 \mathrm{MHz}$ Pentium processor and $2 \mathrm{~GB}$ of memory.

Model Problem Figure 4 illustrates the edge-cracked rectangular bar model used in Sections 4.1 and 4.2. The problem has a single through-the-thickness crack and is discretized with a uniform mesh of $6 *(8 \times 8 \times 4)$ tetrahedral elements. This mesh is created by first generating an $8 \times 8 \times 4$ mesh of hexahedral elements and then dividing each element into 6 tetrahedral elements. Figure 3(a) shows this mesh.

Neumann boundary conditions corresponding to the first term of the Mode I expansion of the elasticity solution in the neighborhood of a crack, $\boldsymbol{u}^{I}$, are applied on the boundary of the global domain as illustrated in Figure 4. The following parameters are assumed: Poisson's ratio $\nu=0.0$; Young's modulus $E=200000$; In-plane dimensions $a=0.5, d=0.875$; Domain thickness $t=1.5$. We set the Poisson's ratio of the material to zero so (i) no tractions need to be applied on the faces $z=0$ and $z=t$ of the domain (ii) the exact solution on this 3-D domain is equal to $\boldsymbol{u}^{I}$.

\subsection{Effect of Boundary Conditions and Size of Local Problems}

Since boundary conditions for local problems are obtained from the solution of an initial (crude) global problem, $\boldsymbol{u}_{G}^{0}$, they are not exact. This can be addressed by $(i)$ improving the accuracy of $\boldsymbol{u}_{G}^{0}$ or (ii) increasing the size of local domains, a procedure commonly used in global-local FEM simulations [14]. These approaches are investigated in Sections 4.1.1 and 4.1.2, respectively.

Three local domain sizes are used: $L_{n r e f=9, \text { nlay }}^{p=(4,4,1)}$ with nlay $=\{1,2,3\}$. The refinement is nonuniform with the elements along the crack front bisected 9 times using the algorithm described 


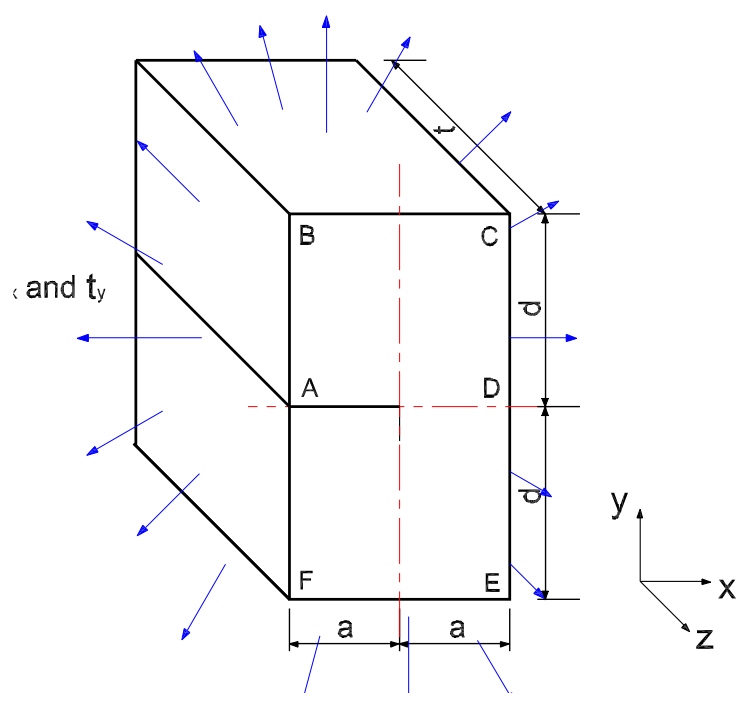

Figure 4. Model problem with a single through-the-thickness crack.

in Section I.1. Three different boundary conditions are applied to the local problems: Dirichlet boundary conditions from the exact solution, $\boldsymbol{u}^{I}$, and from the solutions of global problems $G^{p=(1,1,1)}$ and $G^{p=(4,4,1)}$. The local solutions are then used to enrich nodes located on the crack front of the global problem $G^{p=(4,4,1)}$. The local domains and their construction are illustrated in Figures 5(a) and 5(b). Figure 5(c) illustrates the enriched global problem.

4.1.1. Effect of inexact boundary conditions for local problems Tables I, II, and III list the relative error in the energy norm for the global solution computed from local problems with three different boundary conditions. Here, $N_{l o c}(G)$ denotes the number of degrees of freedom in the local(global) problem, $U_{G}$ denotes the strain energy of the global problem, $e_{G}^{r}$ denotes the relative error in energy norm of the enriched global solution, and $e_{G}^{r \star}$ denotes the relative error in energy norm of the global problem in the case of exact local boundary conditions. On the bottom row of each table, the strain energy and relative error in the energy norm are provided as a reference value when no nodes in the global problem are enriched with the local solution.

From these results, we can observe that the use of global-local enrichment functions greatly enhances the solution of the global problem. The relative error in the global problem is reduced at least $13.79 / 3.77=3.7$ times in all cases after enriching the global problem with local solutions. It can also be seen that only fifteen degrees of freedom are added to the global problem enriched with local solutions since there are five nodes on the global crack front of the model, and three new degrees of freedom are created at each node, which correspond to x-, yand z-components of the local solution.

As expected, the use of local boundary conditions of higher quality improves the quality of enriched global solutions. This improvement is most significant when only a single layer 


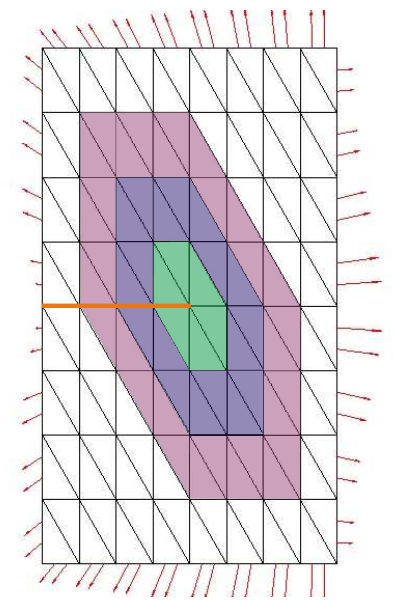

(a) Construction of local discretizations. The shaded area represents the different size of local domains extracted from the coarse global mesh.
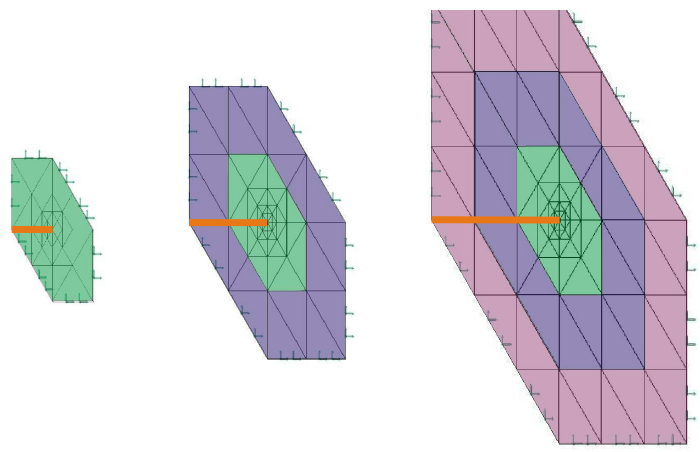

nlay $=1$

$\underline{n l a y}=\mathbf{2}$

$\underline{n l a y}=3$

(b) Local domains for varying number of layers of elements extracted from global mesh.

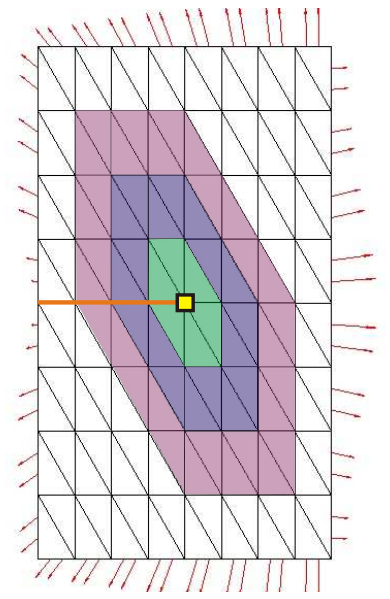

(c) Enrichment of global space with local solutions. Regardless of the local domain size, only the global nodes located on the crack front are enriched with local solutions.

Figure 5. Set-up to analyze the effect of inexact local boundary conditions and local domain size (front view). 
$(n l a y=1)$ is included in the local domain. This can be explained by the St Venant principle. Additional layers in the local domain reduce the effect of the perturbed boundary conditions on the solution near the crack front. However, the difference in $e_{G}^{r}$ computed from the different local boundary conditions is not significant. For example, in case of a single layer in the local domain $($ nlay $=1)$, the global relative error in energy norm $e_{G}^{r}$ obtained from the poor local boundary conditions $\left(G^{p=(1,1,1)}\right)$ is only $8.1 \%$ larger than that of the exact local boundary conditions, $e_{G}^{r \star}$. If we use better local boundary conditions obtained from $G^{p=(4,4,1)}$, the difference is reduced to about $1.3 \%$. This indicates that we can still obtain a good enriched global solution, $\boldsymbol{u}_{G}^{E}$, even if the quality of initial global solution, $\boldsymbol{u}_{G}^{0}$, is not good.

Table I. Effect of inexact boundary conditions for local problems with nlay $=1$ on the error of global problem.

\begin{tabular}{cccccc}
\hline local BCs & $N_{l o c}$ & $N_{G}$ & $U_{G}$ & $e_{G}^{r}(\%)$ & $e_{G}^{r} / e_{G}^{r \star}$ \\
\hline$G^{p=(1,1,1)}$ & 76110 & 12765 & $3.5057446 \mathrm{E}-06$ & 3.7702 & 1.0812 \\
$G^{p=(4,4,1)}$ & 76110 & 12765 & $3.5063528 \mathrm{E}-06$ & 3.5330 & 1.0132 \\
exact & 76110 & 12765 & $3.5064659 \mathrm{E}-06$ & $3.4871\left(=e_{G}^{r \star}\right)$ & 1 \\
\hline No gl-loc enrich. & - & 12750 & $3.4439439 \mathrm{E}-06$ & 13.7930 & - \\
\hline
\end{tabular}

Table II. Effect of inexact boundary conditions for local problems with $n l a y=2$ on the error of global problem.

\begin{tabular}{cccccc}
\hline local BCs & $N_{l o c}$ & $N_{G}$ & $U_{G}$ & $e_{G}^{r}(\%)$ & $e_{G}^{r} / e_{G}^{r \star}$ \\
\hline$G^{p=(1,1,1)}$ & 78060 & 12765 & $3.5058528 \mathrm{E}-06$ & 3.7291 & 1.0607 \\
$G^{p=(4,4,1)}$ & 78060 & 12765 & $3.5063862 \mathrm{E}-06$ & 3.5194 & 1.0010 \\
exact & 78060 & 12765 & $3.5063952 \mathrm{E}-06$ & $3.5158\left(=e_{G}^{r \star}\right)$ & 1 \\
\hline No local sol. & - & 12750 & $3.4439439 \mathrm{E}-06$ & 13.7930 & - \\
\hline
\end{tabular}

Table III. Effect of inexact boundary conditions for local problems with nlay $=3$ on the error of global problem.

\begin{tabular}{cccccc}
\hline local BCs & $N_{\text {loc }}$ & $N_{G}$ & $U_{G}$ & $e_{G}^{r}(\%)$ & $e_{G}^{r} / e_{G}^{r \star}$ \\
\hline$G^{p=(1,1,1)}$ & 80910 & 12765 & $3.5058607 \mathrm{E}-06$ & 3.7260 & 1.0580 \\
$G^{p=(4,4,1)}$ & 80910 & 12765 & $3.5063783 \mathrm{E}-06$ & 3.5226 & 1.0002 \\
exact & 80910 & 12765 & $3.5063801 \mathrm{E}-06$ & $3.5219\left(=e_{G}^{r \star}\right)$ & 1 \\
\hline No local sol. & - & 12750 & $3.4439439 \mathrm{E}-06$ & 13.7930 & - \\
\hline
\end{tabular}

4.1.2. Effect of local domain size The effect of the size of the local domains on the quality of $\boldsymbol{u}_{G}^{E}$ can be quantified using, for example, the data in row 1 of Tables I, II and III. 
The last column of Table IV shows the relative change of global error $e_{G}^{r}$ as the number of layers is increased. Going from one to two layers, for example, gives

$$
\frac{e_{G, \text { nlay }=1}^{r}-e_{G, n l a y=2}^{r}}{e_{G, \text { nlay }=1}^{r}} \times 100=1.0894 \%
$$

The effect of increasing the number of layers, for this class of problems, is therefore minimal. This is due to the smoothness of the exact solution at relatively short distances away from crack fronts as discussed in Section 3.2. As a result, the use of larger local domains is not required. Based on this, hereafter, local problems with only one layer are used.

Table IV. Effect of size of local domains with the initial boundary conditions obtained from $G^{p=(1,1,1)}$ on the error of global problem. The last column shows the relative change of global error $e_{G}^{r}$ as the number of layers is increased from nlay $=1$ to nlay $=2$ and from nlay $=2$ to nlay $=3$.

\begin{tabular}{cccc}
\hline nlay & $N_{l o c}$ & $e_{G}^{r}(\%)$ & Relative reduction of error (\%) \\
\hline 1 & 76110 & 3.7702 & \\
2 & 78060 & 3.7291 & 1.0894 \\
3 & 80910 & 3.7260 & 0.0816 \\
\hline
\end{tabular}

\subsection{Effectiveness of the Proposed Global-Local Approach}

In this section, we investigate how a reduction of the error in the local problems affects the error of a global problem enriched with local solutions. The relation between global and local convergence rates provides us a quantitative measure of the effectiveness of the proposed GFEM with global-local enrichment functions.

Let $\mathcal{I}_{\text {front }}$ denote the index set of the clouds $\omega_{\alpha}$ associated with nodes on the crack front. If the error on all other clouds in the domain is smaller than the error on the clouds $\omega_{\alpha}$, $\alpha \in \mathcal{I}_{\text {front }}$, the estimate for $\left\|\boldsymbol{u}-\boldsymbol{u}_{G}^{E}\right\|_{E\left(\Omega_{G}\right)}$ given in (5) can be written as

$$
\begin{aligned}
\left\|\boldsymbol{u}-\boldsymbol{u}_{G}^{E}\right\|_{E\left(\Omega_{G}\right)} & \leq\left(\sum_{\alpha \in \mathcal{I}_{\text {front }}} \epsilon^{2}(\alpha, \boldsymbol{u})+\sum_{\alpha \notin \mathcal{I}_{\text {front }}} \epsilon^{2}(\alpha, \boldsymbol{u})\right)^{1 / 2} \\
& \leq C\left(\sum_{\alpha \in \mathcal{I}_{\text {front }}} \epsilon^{2}(\alpha, \boldsymbol{u})\right)^{1 / 2}
\end{aligned}
$$

where $C$ is a constant. Thus, if all nodes/clouds in the set $\mathcal{I}_{\text {front }}$ are enriched with global-local enrichment functions, the rate of convergence of the enriched global problem will be controlled by the error of local approximations $\boldsymbol{u}_{h \alpha}, \alpha \in \mathcal{I}_{\text {front }}$, belonging to spaces $\chi_{\alpha}\left(\omega_{\alpha}\right), \alpha \in \mathcal{I}_{\text {front }}$. We can then expect that the solution of the global problem enriched with local solutions will converge at least at the same rate as the local solutions.

Let us consider again the edge-cracked model problem illustrated in Figure 4. The global domain is discretized with a uniform mesh of $6 *(8 \times 8 \times 4)$ tetrahedral elements as before. In the numerical experiments presented in Section 4.2.1 and 4.2.2, the enriched global solution $\boldsymbol{u}_{G}^{E}$ 
is computed on the global discretization $G^{p=(4,4,1)}$. Therefore, the bound provided by (11) and the above observations hold for $\boldsymbol{u}_{G}^{E}$. In Sections 4.2.1 and 4.2.2 we compare the convergence rates of the enriched global problem when the local problems are solved with the $h$ - and $p$-versions of the generalized FEM, respectively. In all numerical experiments, a single local problem is defined for the entire crack front and a single layer of elements is used to define the local domain, i.e., nlay $=1$.

In the convergence analysis presented hereafter, the convergence rates in global and local problems are measured with respect to CPU time, not number of DOFs.

4.2.1. Non-uniform $h$-extensions on the local problem The convergence of the enriched global problem when non-uniform $h$-extensions are performed on the local problems is investigated in this section. Local problems $L_{n r e f, \text { nlay=1 }}^{p=(4,1)}$ with nref $=\{0,3,7,11,15\}$ are solved using three different Dirichlet boundary conditions on $\partial \Omega_{l o c} \backslash\left(\partial \Omega_{l o c} \cap \partial \Omega_{G}\right)$ : The exact solution and the solutions of the global problems $G^{p=(1,1,1)}$ and $G^{p=(4,4,1)}$. For convenience, hereafter these boundary conditions are denoted as 'exact', 'poor' and 'good'. The solutions of these local problems are used as enrichment functions in the global problem $G^{p=(4,4,1)}$ at nodes located at the crack front.

The exact strain energy for the local problem is known when exact boundary conditions are used. However, this is not the case when the boundary conditions are obtained from the solution of the global problem. Hence, in this case, reference values for the exact strain energy is computed using the procedure proposed by Szabo and Babuška [56]. In this procedure, the exact strain energy is estimated using a sequence of finite element solutions and a-priori error estimates for $h$ - or $p$-extensions. The accuracy of the estimated values greatly depends on the accuracy of the approximate solutions. In our computations, the solutions of local problems on discretizations $L_{n r e f}^{p=(4,4,1)}$ with $n r e f=13,14,15$ are used to estimate the exact strain energy for local problems with inexact boundary conditions.

Tables V, VI and VII show the convergence of global and local strain energy when 'poor', 'good' and 'exact' Dirichlet boundary conditions are used on the local problem, respectively. In the tables, $n r e f$ is the level of mesh refinement along the crack front in the local problem, $t_{l o c(G)}$ and $U_{l o c(G)}^{h}$ represent the CPU time spent on the local (global) problem and the computed strain energy for the local (global) problem, respectively. The last column of the tables shows the ratio between the convergence rate in the global and local problems. The rates are measured with respect to CPU time. The exact strain energy for the local and global problems are given in the last row of the tables. The data in the tables are plotted in Figure 6.

The results show that the global convergence rate is always larger than the local convergence rates as predicted by the estimate (11). This behavior is the same for all three cases of boundary conditions applied to the local problem. The ratio between convergence rates decreases with the level of refinement in the local problem but remains larger than one. This decreasing may be due to errors in the global domain away from the crack front. The global problem is enriched only at the crack front. Therefore, as the error around the crack front is reduced, the error elsewhere becomes relevant and the hypothesis used to arrive at estimate (11) is no longer valid.

Figure 6 shows that the error of the enriched global solution is only minimally affected by using a perturbed boundary condition for the local problem instead of the exact boundary condition. Even when local boundary conditions are obtained from a very poor global solution,

[global-local` GFEM`duarte`kim`R1 - August 18, 2007] 
i.e. $G^{p=(1,1,1)}$, the convergence behavior is almost the same as in the case of exact boundary conditions. This demonstrates that the proposed global-local approach to build enrichment functions is very robust.

Table V. Convergence of global and local strain energy. Non-uniform $h$-extensions on local problem with Dirichlet boundary conditions from global problem $G^{p=(1,1,1)}$ ('poor' boundary condition).

\begin{tabular}{cccccc}
\hline nref & $t_{l o c}$ & $t_{G}$ & $U_{l o c}^{h}$ & $U_{G}^{h}$ & Ratio of conv. rates \\
\hline 0 & 1.73 & 65.05 & $3.820950 \mathrm{E}-07$ & $3.471882 \mathrm{E}-06$ & \\
3 & 9.00 & 72.27 & $3.724081 \mathrm{E}-07$ & $3.489150 \mathrm{E}-06$ & 12.51820 \\
7 & 41.57 & 103.25 & $3.685188 \mathrm{E}-07$ & $3.497065 \mathrm{E}-06$ & 3.41673 \\
11 & 197.18 & 186.59 & $3.655763 \mathrm{E}-07$ & $3.503494 \mathrm{E}-06$ & 1.89650 \\
15 & 1022.05 & 401.93 & $3.643902 \mathrm{E}-07$ & $3.506366 \mathrm{E}-06$ & 1.28328 \\
\hline$\infty$ & & & $3.634960 \mathrm{E}-07$ & $3.510735 \mathrm{E}-06$ & \\
\hline
\end{tabular}

Table VI. Convergence of global and local strain energy. Non-uniform $h$-extensions on local problem with Dirichlet boundary conditions from global problem $G^{p=(4,4,1)}$ ('good' boundary condition).

\begin{tabular}{cccccc}
\hline nref & $t_{l o c}$ & $t_{G}$ & $U_{l o c}^{h}$ & $U_{G}^{h}$ & Ratio of conv. rates \\
\hline 0 & 1.84 & 62.84 & $6.931477 \mathrm{E}-07$ & $3.472407 \mathrm{E}-06$ & \\
3 & 8.90 & 71.48 & $6.715684 \mathrm{E}-07$ & $3.489767 \mathrm{E}-06$ & 10.17051 \\
7 & 41.48 & 102.80 & $6.627187 \mathrm{E}-07$ & $3.497709 \mathrm{E}-06$ & 3.50811 \\
11 & 196.84 & 185.95 & $6.560560 \mathrm{E}-07$ & $3.504112 \mathrm{E}-06$ & 2.02149 \\
15 & 1023.74 & 406.67 & $6.533571 \mathrm{E}-07$ & $3.506970 \mathrm{E}-06$ & 1.40798 \\
\hline$\infty$ & & & $6.513262 \mathrm{E}-07$ & $3.510735 \mathrm{E}-06$ & \\
\hline
\end{tabular}

Table VII. Convergence of global and local strain energy. Non-uniform $h$-extensions on local problem with exact Dirichlet boundary condition.

\begin{tabular}{cccccc}
\hline nref & $t_{l o c}$ & $t_{G}$ & $U_{l o c}^{h}$ & $U_{G}^{h}$ & Ratio of conv. rates \\
\hline 0 & 2.12 & 64.52 & $7.994423 \mathrm{E}-07$ & $3.472613 \mathrm{E}-06$ & \\
3 & 9.84 & 73.58 & $7.730479 \mathrm{E}-07$ & $3.489859 \mathrm{E}-06$ & 9.65177 \\
7 & 44.75 & 104.21 & $7.623132 \mathrm{E}-07$ & $3.497808 \mathrm{E}-06$ & 3.63863 \\
11 & 205.14 & 187.58 & $7.541891 \mathrm{E}-07$ & $3.504222 \mathrm{E}-06$ & 2.01493 \\
15 & 1036.79 & 402.39 & $7.508966 \mathrm{E}-07$ & $3.507084 \mathrm{E}-06$ & 1.44377 \\
\hline$\infty$ & & & $7.484435 \mathrm{E}-07$ & $3.510735 \mathrm{E}-06$ & \\
\hline
\end{tabular}

4.2.2. P-extensions on the local problem This section discusses the convergence of the global problem when uniform $p$-extensions are performed on the local problems. Local problems 


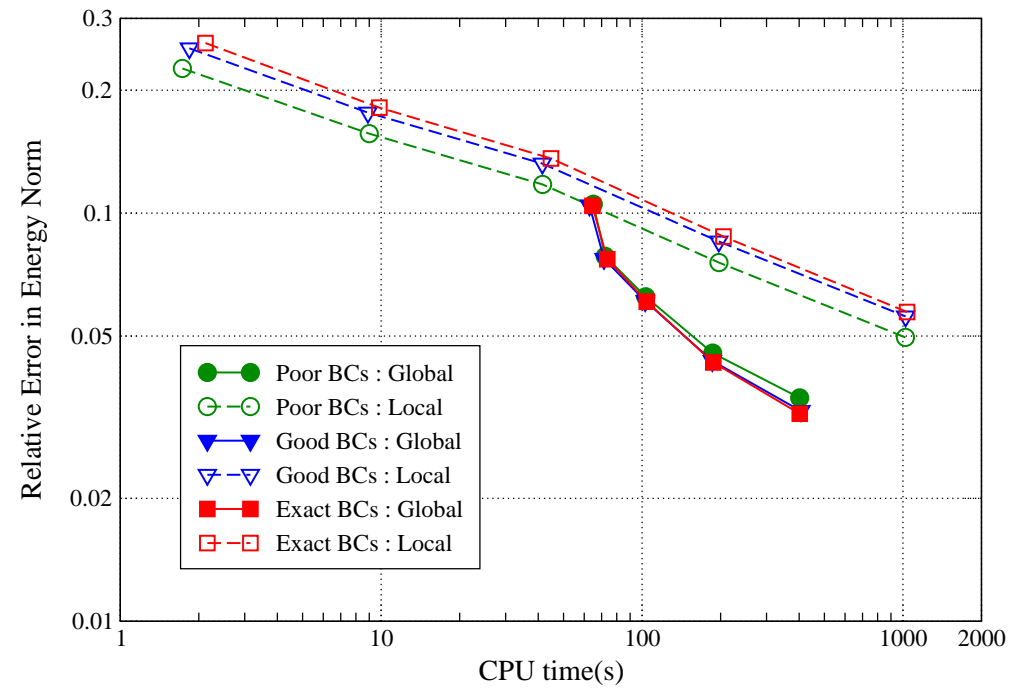

Figure 6. Convergence behavior of global and local problems for non-uniform $h$-extensions on the local problem.

$L_{n r e f=15, \text { nlay }=1}^{p=\left(p_{x}, p_{y}, 1\right)}, 1 \leq p_{x}, p_{y} \leq 4$ are solved using the 'exact', 'poor' and 'good' Dirichlet boundary conditions described in the previous section. The exact and reference values for the strain energy are the same as in the previous section. The solutions of these local problems are used as enrichment functions in the global problem $G^{p=(4,4,1)}$ at nodes located at the crack front. Note that 15 levels of mesh refinement are applied to the local problems in order to isolate the singularity at the crack front. Tables VIII, IX and X and Figure 7 present the results. Similar to the non-uniform $h$-extensions case, only a small difference is found between global solutions enriched with local solutions computed with 'exact', 'good' or 'poor' boundary conditions.

Table VIII. Convergence of global and local strain energy. $P$-extension on local problem with Dirichlet boundary conditions from global problem $G^{p=(1,1,1)}$ ('poor' boundary condition).

\begin{tabular}{cccccc}
\hline$p$-order. & $t_{l o c}$ & $t_{G}$ & $U_{l o c}^{h}$ & $U_{G}^{h}$ & Ratio of conv. rates \\
\hline 1 & 2.15 & 293.07 & $3.912982 \mathrm{E}-07$ & $3.481001 \mathrm{E}-06$ & \\
2 & 32.09 & 320.41 & $3.687048 \mathrm{E}-07$ & $3.500050 \mathrm{E}-06$ & 18.52115 \\
3 & 226.95 & 359.76 & $3.653164 \mathrm{E}-07$ & $3.504406 \mathrm{E}-06$ & 8.41271 \\
4 & 1022.05 & 401.93 & $3.643902 \mathrm{E}-07$ & $3.506366 \mathrm{E}-06$ & 7.07854 \\
\hline$\infty$ & & & $3.634960 \mathrm{E}-07$ & $3.510735 \mathrm{E}-06$ & \\
\hline
\end{tabular}


Table IX. Convergence of global and local strain energy. P-extension on local problem with Dirichlet boundary conditions from global problem $G^{p=(4,4,1)}$ ('good' boundary condition).

\begin{tabular}{cccccc}
\hline$p$-order. & $t_{l o c}$ & $t_{G}$ & $U_{l o c}^{h}$ & $U_{G}^{h}$ & Ratio of conv. rates \\
\hline 1 & 2.36 & 292.65 & $7.299275 \mathrm{E}-07$ & $3.481729 \mathrm{E}-06$ & \\
2 & 32.40 & 320.35 & $6.613327 \mathrm{E}-07$ & $3.500799 \mathrm{E}-06$ & 15.05567 \\
3 & 228.51 & 355.63 & $6.550298 \mathrm{E}-07$ & $3.505020 \mathrm{E}-06$ & 10.40229 \\
4 & 1023.74 & 406.67 & $6.533571 \mathrm{E}-07$ & $3.506970 \mathrm{E}-06$ & 7.77007 \\
\hline$\infty$ & & & $6.513262 \mathrm{E}-07$ & $3.510735 \mathrm{E}-06$ & \\
\hline
\end{tabular}

Table X. Convergence of global and local strain energy. $P$-extension on local problem with exact Dirichlet boundary condition.

\begin{tabular}{cccccc}
\hline$p$-order. & $t_{l o c}$ & $t_{G}$ & $U_{l o c}^{h}$ & $U_{G}^{h}$ & Ratio of conv. rates \\
\hline 1 & 2.44 & 294.16 & $8.435237 \mathrm{E}-07$ & $3.481771 \mathrm{E}-06$ & \\
2 & 33.68 & 323.18 & $7.611729 \mathrm{E}-07$ & $3.500909 \mathrm{E}-06$ & 14.99954 \\
3 & 234.94 & 356.55 & $7.531670 \mathrm{E}-07$ & $3.505132 \mathrm{E}-06$ & 11.20100 \\
4 & 1036.79 & 402.39 & $7.508966 \mathrm{E}-07$ & $3.507084 \mathrm{E}-06$ & 8.02238 \\
\hline$\infty$ & & & $7.484435 \mathrm{E}-07$ & $3.510735 \mathrm{E}-06$ & \\
\hline
\end{tabular}

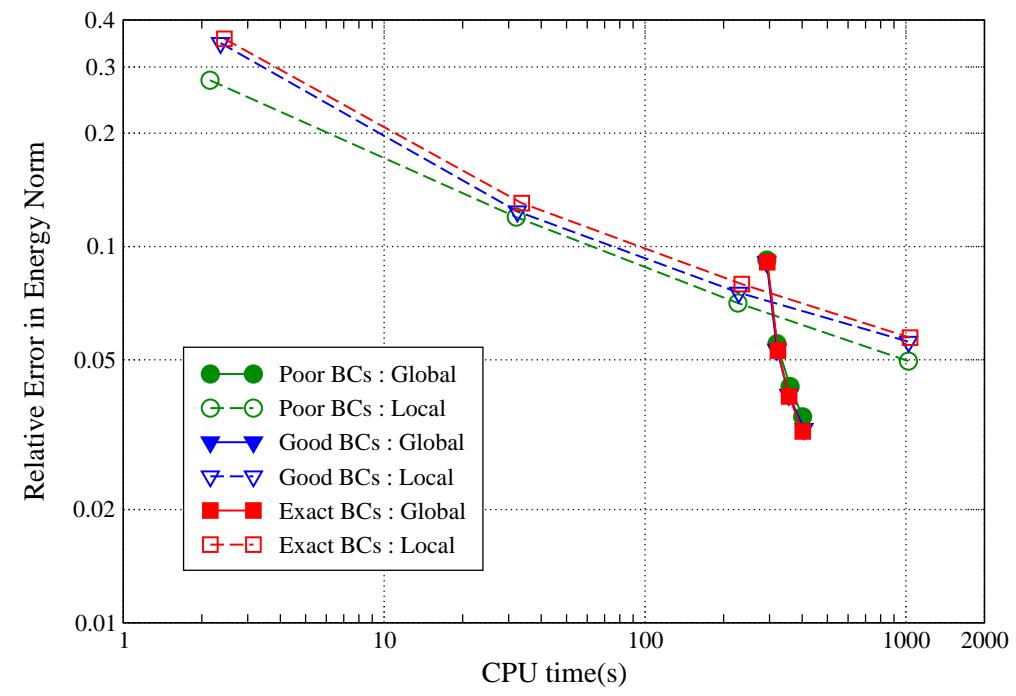

Figure 7. Convergence behavior of global and local problems for $p$-extensions on the local problem. 


\subsection{Cost Analysis of the GFEM with Global-Local Enrichment Functions}

The GFEM with global-local enrichment functions can potentially be more efficient than the FEM or the standard GFEM (i.e., the GFEM without global-local enrichment functions). This may be the case because the computational cost of solving a linear system grows faster than linearly with respect to problem size. Therefore, it may be more computationally efficient to solve several smaller local problems instead of a single, large, global problem. Another advantage of solving several local problems instead of a single large problem, is the reduced memory requirements. The amount of memory required to solve a local problem is much smaller than that required to solve a single large global problem and it can be released after computing the local solution vector. Therefore, the GFEM with global-local enrichment functions also has the potential of solving, on a given hardware, larger problems than, e.g., the FEM. Memory usage is specially critical on 32-bit hardware. On the other hand, the numerical integration of global-local enrichment functions is more involved than in the standard GFEM or in the FEM, as discussed in Section I.3. This may offset the gains of the method during the factorization procedure of the stiffness matrix.

Some of the computational issues discussed above are investigated in this section. Specifically, we investigate the computational cost of the GFEM with global-local enrichment functions when solving stationary cracks on a serial computer. In the analysis, we consider the effect of the number of local problems on the performance of the method. A model problem with one, three or five cracks is analyzed. We compare the computational cost of the GFEM with global-local enrichment functions with the standard GFEM. In the latter, the accuracy of the solution of controlled through mesh refinements of the global mesh (like in the FEM), while in the former the global mesh is fixed and the accuracy is controlled through global-local enrichment functions only. In both methods, only polynomial enrichment functions and meshes fitting the crack surface, as in the FEM, are used. In other words, a situation like that shown in Figure 1, with a crack modeled with discontinuous and/or singular enrichment functions, is not considered. The standard GFEM used here is therefore very similar to the FEM. Hereafter, we denote the GFEM with global-local enrichment functions simply by $G F E M^{\mathrm{g}-1}$. The reported $\mathrm{CPU}$ time for the FFEM $^{\mathrm{g}-\mathrm{l}}$ includes both local and global computations.

Model Problem A model problem with up to five cracks and illustrated in Figure 8 is used in the computations below. In the computations, only half of the domain (domain CDFG in Figure 8 ) is discretized and symmetry boundary conditions applied along the vertical plane of symmetry. The domain is discretized with a mesh of $6 *(6 \times 12 \times 1)$ tetrahedral elements. This mesh is used for the global computations in the $G F E M^{\mathrm{g}-1}$ and as an initial mesh for the standard GFEM. Examples of discretizations used in both approaches are shown in Figures 10 and 11. The exact strain energy for this problem was estimated using the procedure described in Section 4.2.1.

The following parameters are assumed for this model: Poisson's ratio $\nu=0.33$; Young's modulus $E=200000$; In-plane dimensions $d=36.0, a_{1}=18.0, a_{2}=12.0, a_{3}=6.0$, $b_{1}=b_{2}=6.0$; Domain thickness $t=6.0$; Neumann boundary conditions

$$
\begin{aligned}
& t_{x}=0.0, t_{y}=20.0, t_{z}=0.0 \quad \text { on the top side of the body } \\
& t_{x}=0.0, t_{y}=-20.0, t_{z}=0.0 \quad \text { on the bottom side of the body }
\end{aligned}
$$

A non-zero Poisson's ratio is used in this problem. Therefore, a triaxial stress state develops

[global-local' GFEM'duarte 'kim`R1 - August 18, 2007] 


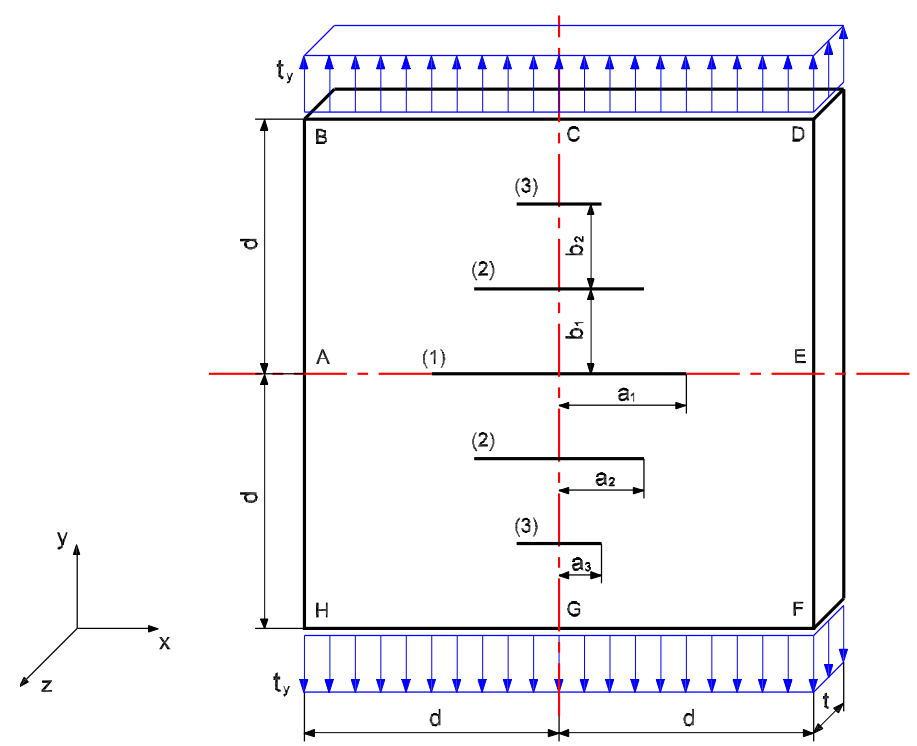

Figure 8. Multiple crack example. Possible crack locations are indicated as (1),(2) and (3).

near the crack fronts. This three-dimensional effect is more pronounced near the intersection of a crack front and the boundary of the domain (at the crack front vertices). Figure 9 shows the Euclidian norm of the solution near the crack front. Three-dimensional effects can be observed. This behavior is accounted for by further refining the meshes around the crack front vertices. The levels of refinement at the crack fronts and their vertices are listed on Tables XI and XII for the case of the model with five cracks. They are indicated as "F" and "V", respectively, in the tables.

Standard GFEM The discretization error in this case is controlled through non-uniform mesh refinements in the neighborhood of the crack fronts. Examples of discretizations used are shown in Figure 10. Global discretizations with polynomial order $p=(5,5,5)$ and increasing levels of mesh refinement around the crack fronts are used.

GFEM with global-local enrichments The procedure used in the $G F E M^{\mathrm{g}-\mathrm{l}}$ is illustrated in Figure 11. The mesh refinement in the local problems is non-uniform and graded towards the crack front. Figure 11(b) illustrates a local discretization.

The initial global solution, $\boldsymbol{u}_{G}^{0}$, is computed on the global discretization $G^{p=(5,5,5)}$. This solution provides Dirichlet boundary conditions for the local problems as described in Section 3.2. The local problems are solved on discretizations $L_{n r e f, \text { nlay }=1}^{p=(5,5,5)}$. The local solutions are used as enrichment functions for the global problem $G^{p=(5,5,5)}$. 


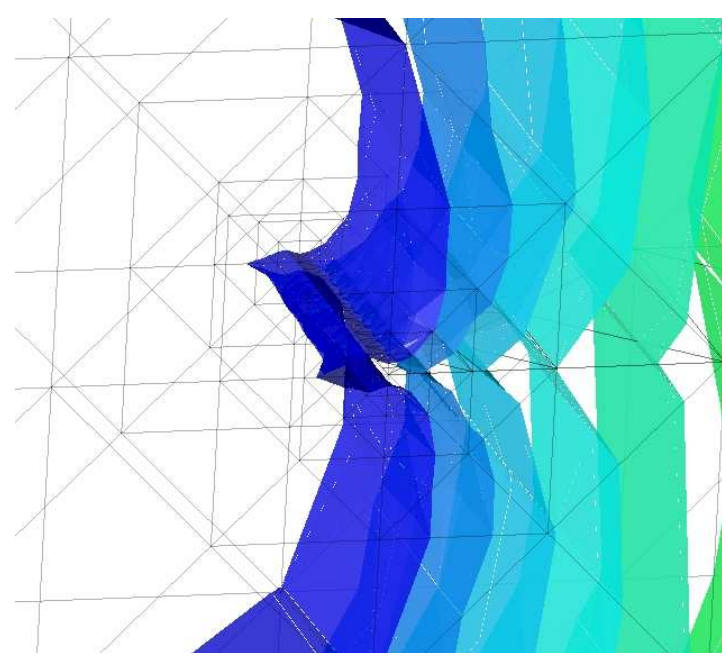

Figure 9. Isosurface of the Euclidian norm of the solution near the crack front. Three-dimensional effects can be observed.

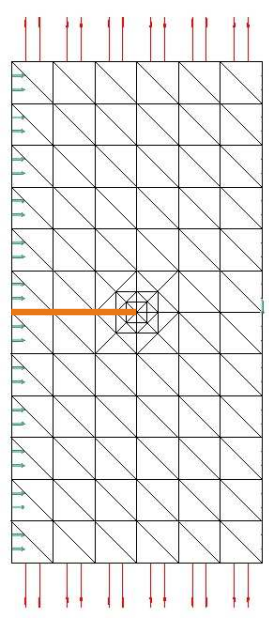

$\underline{\text { single crack }}$

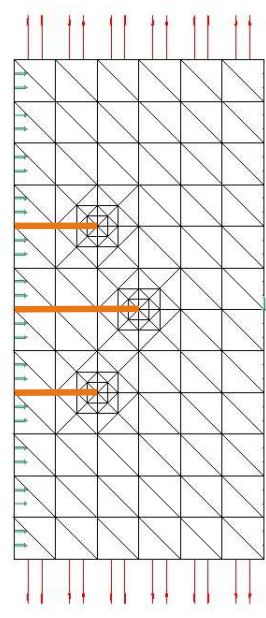

3 cracks

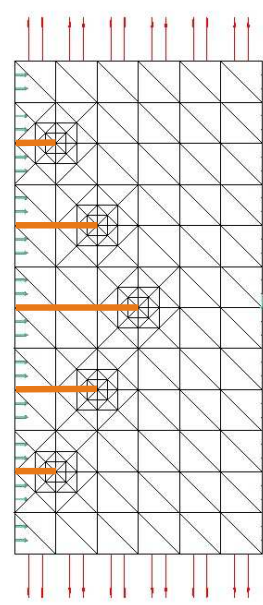

$\underline{5 \text { cracks }}$

Figure 10. Analysis of the domain with multiple cracks by the standard GFEM (top view). Nonuniform $h$-refinement is performed on the elements near each crack front. 


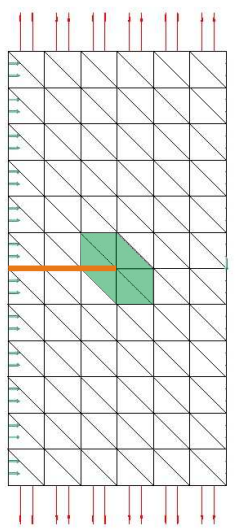

single crack

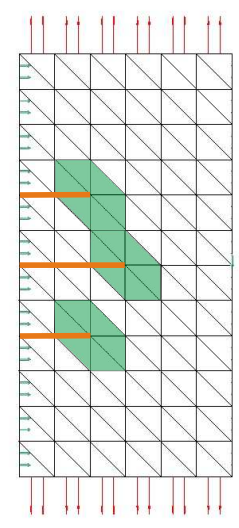

3 cracks

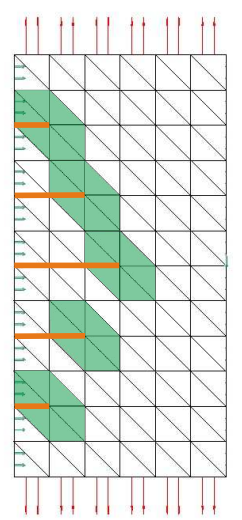

$\underline{5 \text { cracks }}$

(a) Construction of local discretizations. The shaded area represents the local domain extracted from the coarse global mesh.

(b) Graded mesh used in the discretization of a local problem.

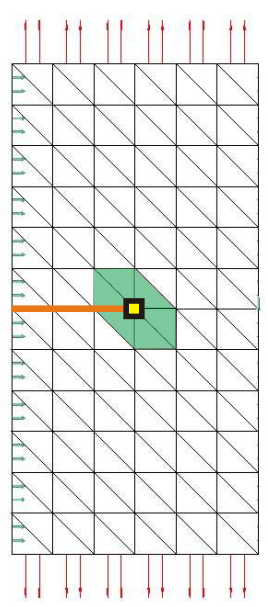

single crack

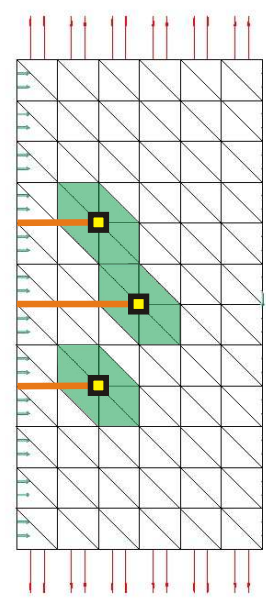

3 cracks

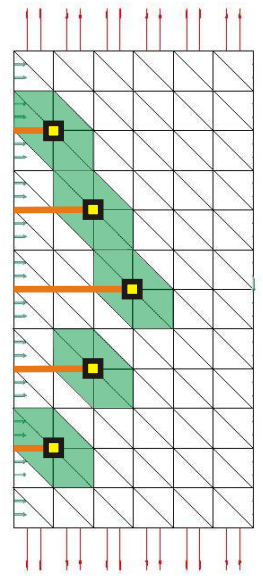

5 cracks

(c) Enrichment of global discretization with local solutions. Yellow squares on the crack fronts represent the nodes enriched with the local solutions.

Figure 11. Analysis of the domain with multiple cracks by the $G F E M^{\mathrm{g}-1}$ (top view). Mesh refinements are applied to local problems only. 
In the numerical experiments presented in this section, the maximum level of $h$-refinement is limited by the maximum amount of memory a 32 bit machine can allocate. As a result, each example shows a different level of maximum $h$-refinement. The $G F E M^{\text {g-l }}$ can reach a higher level of mesh refinement in the local problems than the standard GFEM can in the global problem, as discussed before.

The results for the model with five cracks solved with GFEM $^{\mathrm{g}-\mathrm{l}}$ and standard GFEM are presented in Tables XI and XII, respectively. The tables list the level of refinement (nref) at crack fronts (F) and additional refinement level performed at each crack vertex (V), the number of degrees of freedom $(N)$, the relative error in the energy norm in the enriched global domain $\left(e_{G}^{r}\right)$ and the CPU time spent on the assembly $\left(t_{\text {assem }}\right)$, factorization $\left(t_{\text {fact }}\right)$ and backward and forward substitution $\left(t_{s u b}\right)$ of the stiffness matrix in both local and global problems. They also report $t_{t o t}$, which measures the total CPU time spent in a $G F E M^{\mathrm{g}-1}$ or in a standard GFEM analysis. In the case of a $G F E M^{\text {g-l }}$ analysis, $t_{t o t}$ includes the CPU time spent in the local problems. The three essential components which comprise most of the computational cost of the analysis, are $t_{\text {assem }}, t_{\text {fact }}$ and $t_{\text {sub }}$. The local $t_{\text {assem }}, t_{\text {fact }}$ and $t_{\text {sub }}$ reported in Table XI, are the total CPU time spent in all five local problems while $N$ local is for each local problem.

From the results reported in the tables, some unique features of the CPU time spend in a $G F E M^{\mathrm{g}-1}$ analysis can be identified. As the number of degrees of freedom in a local problem increases (as the local domain is $h$-refined), $t_{\text {assem }}, t_{\text {fact }}$ and $t_{s u b}$ of the local problems increase, whereas only $t_{\text {assem }}$ increases in the global problem. This is because the number of degrees of freedom in the global problem does not depend on the size of the local problems. Comparing the first and last rows of Table XI, we can observe that the error of the enriched global solution was reduced to about one third of the initial error using the same number of degrees of freedom in the global problem. The assembly time in the global problem, $t_{\text {assem }}$, increases since mesh refinement in the local problems requires more integration points in the global elements enriched with local solutions, as described in Section I.3. Note however, that $t_{\text {assem }}$ in the global problem grows much slower than the total $t_{a s s e m}$ in the local problems. This attests that the exact integration procedure described in Section I.3 can be efficiently implemented.

Table XI. CPU time spent on the model with five cracks shown in Figure 11 using the GFEM ${ }^{\mathrm{g}-1}$. In the local problem, $N$ is the number of degrees of freedom used in each local problem while the CPU times reported account for all five local problems. $t_{t o t}$ measures the total CPU time spent in a FFEM $^{\mathrm{g}-1}$ analysis and $e_{G}^{r}$ is the relative error in energy norm of the enriched global solution.

\begin{tabular}{|c|c|c|c|c|c|c|c|c|c|c|}
\hline \multirow{2}{*}{$\begin{array}{c}\text { nref } \\
(\mathrm{F} / \mathrm{V})\end{array}$} & \multicolumn{4}{|c|}{ Local problems } & \multicolumn{4}{|c|}{ Global problem } & \multirow[b]{2}{*}{$t_{t o t}$} & \multirow[b]{2}{*}{$e_{G}^{r}$} \\
\hline & $N$ & $t_{\text {assem }}$ & $t_{\text {fact }}$ & $t_{\text {sub }}$ & $N$ & $t_{\text {assem }}$ & $t_{\text {fact }}$ & $t_{\text {sub }}$ & & \\
\hline$(0 / 0)$ & 1680 & 12.8 & 16.8 & 0.2 & 20580 & 47.4 & 49.8 & 3.2 & 130.2 & 0.09169 \\
\hline$(1 / 1)$ & 4620 & 63.7 & 97.0 & 0.9 & 20580 & 80.9 & 49.8 & 3.2 & 295.5 & 0.06255 \\
\hline$(2 / 2)$ & 8925 & 158.8 & 220.9 & 2.3 & 20580 & 143.7 & 50.2 & 3.2 & 579.1 & 0.05230 \\
\hline$(3 / 3)$ & 14490 & 285.2 & 566.0 & 4.0 & 20580 & 222.9 & 49.7 & 3.2 & 1130.9 & 0.04384 \\
\hline$(4 / 4)$ & 20580 & 458.0 & 2225.2 & 7.3 & 20580 & 330.3 & 49.7 & 3.2 & 3073.7 & 0.03882 \\
\hline$(5 / 5)$ & 32235 & 721.2 & 3890.8 & 11.6 & 20580 & 497.3 & 49.7 & 3.2 & 5173.7 & 0.03260 \\
\hline$(6 / 5)$ & 38325 & 923.5 & 7020.2 & 22.0 & 20580 & 617.3 & 49.6 & 3.2 & 8635.8 & 0.03101 \\
\hline
\end{tabular}

The relative error in energy norm versus CPU time is plotted in Figure 12 for computations 
Table XII. CPU time spent on the model with five cracks shown in Figure 10 using standard GFEM.

\begin{tabular}{c||cccc|c||c}
\hline $\begin{array}{c}\text { nref } \\
(\mathrm{F} / \mathrm{V})\end{array}$ & $N$ & $t_{\text {assem }}$ & $t_{\text {fact }}$ & $t_{\text {sub }}$ & $t_{\text {tot }}$ & $e^{r}$ \\
\hline$(0 / 0)$ & 21000 & 50.0 & 56.8 & 3.4 & 110.2 & 0.10612 \\
$(1 / 1)$ & 35700 & 105.4 & 176.3 & 6.8 & 288.5 & 0.07175 \\
$(2 / 2)$ & 57225 & 200.1 & 389.4 & 12.6 & 602.1 & 0.05485 \\
$(3 / 2)$ & 66675 & 256.8 & 694.8 & 17.5 & 969.1 & 0.05125 \\
\hline
\end{tabular}

on domains with one, three and five cracks. The convergence plots are for the standard and $G F E M^{\mathrm{g}-1}$ when non-uniform $h$-extensions are performed at the global and local discretizations, respectively. Several observations can be made based on this plot.

First, for any number of cracks in the domain, there is a range of discretization error and CPU time over which the $G F E M^{\mathrm{g}-1}$ is more computational efficient than the standard GFEM. The magnitude of this range increases with the number of cracks/local problems in the domain. This indicates that the $G F E M^{\mathrm{g}-1}$ can be quite more efficient than the standard GFEM or the FEM when analyzing problems with several cracks or other local features in the domain. It also indicates that the efficiency of the GFEM ${ }^{\mathrm{g}-\mathrm{l}}$ compared to the standard GFEM can be extended by increasing the number of local problems. For example, by creating more than one local problem at each crack front.

Second, the $G F E M^{\mathrm{g}-1}$ approach is less affected by hardware limitations than the standard GFEM. The last point in each curve shown in Figure 12, represents the maximum level of nonuniform $h$-refinement which could be solved in a 32 bit machine. As a result, the $G F E M^{\mathrm{g}-1}$ can deliver more accurate solutions on a given hardware than the standard GFEM. In the five crack model, the most accurate solution computed with the standard GFEM, has an error that is about $65 \%$ larger than the most accurate solution the $G F E M^{\mathrm{g}-1}$ was able to compute.

Third, we observe a reduction in the convergence rate with mesh refinement in both the standard and $G F E M^{\mathrm{g}-1}$. This decreasing is due to errors in the global domain away from the crack fronts. In both methods, the meshes are refined only near the crack fronts. Therefore, as the error around these regions is reduced, the error elsewhere in the domain becomes relevant. The reduction in the convergence rate is less pronounced in case of the standard GFEM since the mesh refinement applied to the elements at the crack front induces some additional refinement required to keep the mesh conforming. This propagation of mesh refinement does not happen in the GFEM ${ }^{\mathrm{g}-1}$ approach. This can be verified by comparing the mesh refinements near the crack fronts in Figures 10 and 11(b).

From the above, we can conclude that even on a serial computer and for stationary cracks, the $G F E M^{g-1}$ can be a very effective tool to analyze a problem with many cracks or other local features.

\section{Summary and Concluding Remarks}

In this paper, we analyze a two-level approach to build enrichment functions for partition of unity methods and, in particular, for the generalized FEM. Three-dimensional fracture 


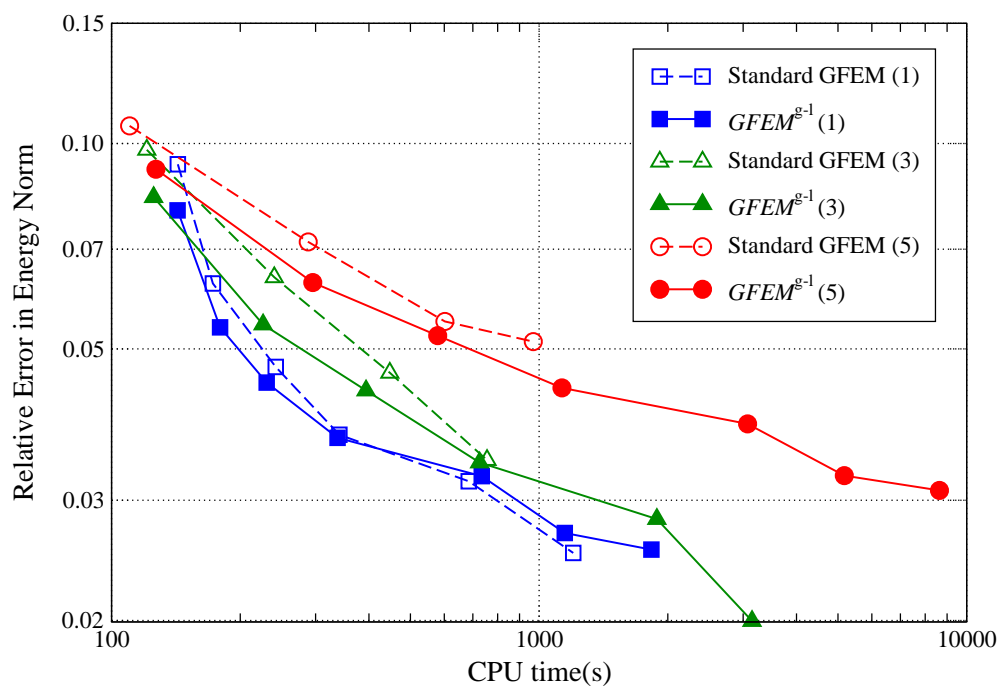

Figure 12. Effect of number of local problems on the performance of the $G F E M^{g-1}$. The number enclosed by parentheses in the legend represents the number of cracks in the global domain.

mechanics problems are used to illustrate the main ideas of the procedure. The approach is, however, not limited to this particular application.

The main features of the generalized FEM with global-local enrichment functions are summarized below.

- The procedure accounts for possible interactions of local (near crack for example) and global (structural) behavior. This is in contrast with standard global-local FEM [37] which is broadly used in many engineering applications of the FEM;

- Local features, like crack fronts, are several orders of magnitude smaller than the size of the domain of interest (a complex structure, for example). The mesh density required in the neighborhood of a crack front is several orders of magnitude larger than in parts of the domain with smooth solutions. As a result, mesh refinements at the crack front usually propagate beyond the region of interest in order to create a conforming global mesh. In the GFEM ${ }^{\mathrm{g}-1}$, this problem does not exist since the refinement is done only in the local domains. Therefore, no matter how refined the local domains are, no refinement is performed in the, usually large, global problem.

The ability to transition between discretizations with arbitrarily distinct mesh densities is also important when dealing with multiscale problems.

Other approaches used to incorporate a very refined mesh into a coarse one are, for example, mortar elements and Lagrange Multipliers [11, 28, 42, 57]. Lagrange multiplier methods [2] lead to a saddle point problem and the Babuška-Brezzi condition [12, 38] must be satisfied for stability;

- The computation at local problems can be parallelized without difficulty allowing the solution of large problems very efficiently. This feature of the methodology is related to the various domain decomposition techniques available in the literature $[9,11,13,46,48]$;

- The size of the global problems solved with the proposed approach is almost independent 
of the resolution used to resolve the local features. This happens because the number of degrees of freedom added to the global problem does not depend on the number of degrees of freedom used in the local problems. It depends only on the number of global nodes enriched with local solutions; This is in contrast with the s-version of the FEM $[27,31,43]$ in which shape functions from local meshes superposed on a global mesh are used in the solution of the global problem;

- The computation of the solution of the enriched global problem, $\boldsymbol{u}_{G}^{E}$, can be done very efficiently once the coarse global problem has been solved (Cf. Section I.2). This can lead to substantial savings in computations when solving, for example, crack propagation or time dependent problems;

- In this paper, local problems are solved with the GFEM. However, it is possible to utilize other approaches and paste the local solutions using the partition of unity of the global problem as presented here.

The main conclusions of this analysis presented in this paper are as follows:

- The integration of global-local enrichment functions can be done exactly using the meshes of the local problems which are nested in the global mesh. The numerical experiments presented in Section 4.3 show that this procedure can be efficiently implemented;

- Boundary conditions for local problems are obtained from the solution of an initial (crude) global problem, $\boldsymbol{u}_{G}^{0}$. In Section 4.1.1 we demonstrate that the effect of these inexact boundary conditions on the accuracy of the enriched global solution, $\boldsymbol{u}_{G}^{E}$, is not significant.

- The numerical experiments presented in Section 4.1.2 show that the quality of the enriched global solution, $\boldsymbol{u}_{G}^{E}$, is also not significantly affected by the size of the local domains. This allows us to use local domains with only one layer of elements around crack fronts;

- The information transfer between local (fine) and global (coarse) scales using the partition of unity framework is very effective, as demonstrated in Section 4.2. We show that the global problem converges at least as fast as the local problems;

- A detailed cost analysis of the GFEM with global-local enrichment functions is presented in Section 4.3. The numerical experiments show that even when analyzing stationary cracks on a serial computer, the GFEM with global-local enrichment functions has the potential to be more efficient than the FEM or the standard GFEM.

\section{APPENDIX}

\section{Computational Implementation}

In this appendix, we present some details of the numerical implementation of the proposed GFEM with global-local enrichment functions.

\section{I.1. Construction of Local Discretizations}

Discretizations for local problems defined in a neighborhood of a crack front are constructed as follows. We restrict the definitions to the case of a global discretization with crack fronts 
located along element boundaries, like the case shown in Figure 3(a). A situation like that shown in Figure 1, in which the crack front is arbitrarily located in the mesh, can be dealt with analogously. We also assume that a single local problem is defined for each crack in the domain. Extensions to multiple, smaller, local problems per crack are not difficult. The effectiveness of using more than one local problem per crack front and their parallel solution is the subject of our future work.

Let $\mathcal{I}_{\text {front }}$ denote the indices of all nodes from the global mesh located along crack front $\Gamma_{\text {front }}$. Local meshes are created using elements extracted from the global mesh around the crack front. A local domain corresponding to a mesh with one layer of elements around the crack front is given by

$$
\Omega_{\text {loc }}^{\text {nlay }=1}:=\bigcup_{\beta \in \mathcal{I}_{\text {front }}} \omega_{\beta}
$$

where $\omega_{\beta}$ is the union of (copy of) global elements sharing vertex node $\boldsymbol{x}_{\beta}, \beta \in \mathcal{I}_{\text {front }}$. Note that $\Omega_{\text {loc }}^{\text {nlay=1}}$ contains the entire crack front. Local domains with additional layers of elements around the crack front are defined analogously. The mesh corresponding to a local domain with $m$ layers of elements around the crack front is given by the union of (copy of) the mesh with $m-1$ layers and global elements sharing a vertex node in the mesh with $m-1$ layers. Figure 3(b) illustrates a local domain extracted from the global mesh shown in Figure 3(a).

Local Refinement Around a Crack Front A local discretization with one level of mesh refinement $\left(L_{\text {nref }=1, n l a y}^{p}\right)$ around a crack front is performed by first bisecting, in the initial mesh, all elements with nodes on the crack front and then bisecting additional elements in order to recover a conforming discretization. The marked-edge algorithm $[1,8]$ is used to select the refinement edges of the elements. This procedure is repeated $n-1$ times for a refinement level $n r e f=n$. The initial mesh extracted from the global mesh corresponds to nref $=0$. Figure 3 (c) shows an example of the application of this algorithm to the local mesh shown in Figure 3 (b). This local mesh refinement algorithm preserves the nesting of local elements into the global mesh. This greatly facilitates the computational implementation and provides many opportunities for optimization of the code.

\section{I.2. Solution of Enriched Global Problem}

In the last step of the proposed GFEM, the global problem is enriched with the solution of a local problem using Equation (10). The solution of the local problem shown in Figure 3(d), $\boldsymbol{u}_{l o c}$, is used as an enrichment function for the global (coarse) problem shown in Figure 3(a). A large number of degrees of freedom may be used in the computation of $\boldsymbol{u}_{l o c}$. However, only three degrees of freedom are added to each global node in the coarse mesh enriched with $\boldsymbol{u}_{l o c}$, when solving the elasticity equations in three dimensions. In addition, the degrees of freedom associated with global-local enrichments are hierarchically added to the initial global problem, i.e., all initial global shape functions remain unmodified in the global problem. Several approaches can take advantage of these properties of the enriched global problem and, as demonstrated below, the computation cost of the enriched global global solution $\boldsymbol{u}_{G}^{E}$ is small. At least two approaches can be used to efficiently compute $\boldsymbol{u}_{G}^{E}$ :

(i) Iterative solvers designed for a sequence of linear systems [44];

[global-local'GFEM`duarte 'kim`R1 - August 18, 2007] 
(ii) Re-use of the factorization of the global matrix associated with the initial (coarse) global problem.

In this section, we illustrate the last approach for the case of a linear quasi-static problem solved with a direct solver. The procedure involves standard matrix partitioning and condensation of degrees of freedom [58]. Extensions to non-linear problems are also possible, since in this case the problem can be solved as a sequence of linear problems.

Let

$$
\boldsymbol{K}_{G}^{0} \underline{\boldsymbol{u}}_{G}^{0}=\boldsymbol{F}_{G}^{0}
$$

and

$$
\boldsymbol{K}_{G}^{E} \underline{\boldsymbol{u}}_{G}^{E}=\boldsymbol{F}_{G}^{E}
$$

denote the systems of equations associated with the initial (crude) and enriched global discretizations, respectively ${ }^{1}$. Vectors $\underline{\boldsymbol{u}}_{G}^{E}$ and $\boldsymbol{F}_{G}^{E}$ can be partitioned, using the hierarquical property of the enrichment functions, as follows

$$
\begin{aligned}
& \underline{\boldsymbol{u}}_{G}^{E}=\left[\begin{array}{ll}
\tilde{\boldsymbol{u}}_{G}^{0} & \underline{\boldsymbol{u}}_{G}^{\mathrm{g}-1}
\end{array}\right]^{T} \\
& \boldsymbol{F}_{G}^{E}=\left[\begin{array}{ll}
\boldsymbol{F}_{G}^{0} & \boldsymbol{F}_{G}^{\mathrm{g}-1}
\end{array}\right]^{T}
\end{aligned}
$$

where $\underline{\tilde{u}}_{G}^{0}$ are degrees of freedom associated with the initial (coarse) global discretization and $\underline{\boldsymbol{u}}_{G}^{\mathrm{g}-\mathrm{l}}$ are degrees of freedom associated with global-local hierarchical enrichments. The enriched global matrix can then be written as

$$
\left[\begin{array}{ll}
\boldsymbol{K}_{G}^{0} & \boldsymbol{K}_{G}^{0, g-1} \\
\boldsymbol{K}_{G}^{\mathrm{g}-1,0} & \boldsymbol{K}_{G}^{\mathrm{g}-1}
\end{array}\right]\left[\begin{array}{l}
\underline{\tilde{\boldsymbol{u}}}_{G}^{0} \\
\underline{\boldsymbol{u}}_{G}^{\mathrm{g}-1}
\end{array}\right]=\left[\begin{array}{l}
\boldsymbol{F}_{G}^{0} \\
\boldsymbol{F}_{G}^{\mathrm{g}-1}
\end{array}\right]
$$

where $\boldsymbol{K}_{G}^{0, \mathrm{~g}-\mathrm{l}}=\left(\boldsymbol{K}_{G}^{\mathrm{g}-1,0}\right)^{T}$.

From the first equation above we have

$$
\underline{\tilde{\boldsymbol{u}}}_{G}^{0}=\left(\boldsymbol{K}_{G}^{0}\right)^{-1}\left[\boldsymbol{F}_{G}^{0}-\boldsymbol{K}_{G}^{0, \mathrm{~g}-1} \underline{\boldsymbol{u}}_{G}^{\mathrm{g}-1}\right]
$$

Substituting the above in the second equation of (12)

$$
\begin{gathered}
\boldsymbol{K}_{G}^{\mathrm{g}-1,0}\left(\boldsymbol{K}_{G}^{0}\right)^{-1}\left[\boldsymbol{F}_{G}^{0}-\boldsymbol{K}_{G}^{0, \mathrm{~g}-1} \underline{\boldsymbol{u}}_{G}^{\mathrm{g}-1}\right]+\boldsymbol{K}_{G}^{\mathrm{g}-1} \underline{\boldsymbol{u}}_{G}^{\mathrm{g}-1}=\boldsymbol{F}_{G}^{\mathrm{g}-1} \\
{\left[\boldsymbol{K}_{G}^{\mathrm{g}-1}-\boldsymbol{K}_{G}^{\mathrm{g}-1,0}\left(\boldsymbol{K}_{G}^{0}\right)^{-1} \boldsymbol{K}_{G}^{0, \mathrm{~g}-1}\right] \underline{\boldsymbol{u}}_{G}^{\mathrm{g}-1}=\boldsymbol{F}_{G}^{\mathrm{g}-1}-\boldsymbol{K}_{G}^{\mathrm{g}-1,0}\left(\boldsymbol{K}_{G}^{0}\right)^{-1} \boldsymbol{F}_{G}^{0}} \\
\widehat{\boldsymbol{K}}_{G}^{\mathrm{g}-1} \underline{\boldsymbol{u}}_{G}^{\mathrm{g}-1}=\widehat{\boldsymbol{F}}_{G}^{\mathrm{g}-1}
\end{gathered}
$$

where

$$
\widehat{\boldsymbol{K}}_{G}^{\mathrm{g}-1}=\left[\boldsymbol{K}_{G}^{\mathrm{g}-1}-\boldsymbol{K}_{G}^{\mathrm{g}-1,0}\left(\boldsymbol{K}_{G}^{0}\right)^{-1} \boldsymbol{K}_{G}^{0, \mathrm{~g}-1}\right]
$$

and

$$
\widehat{\boldsymbol{F}}_{G}^{\mathrm{g}-1}=\boldsymbol{F}_{G}^{\mathrm{g}-1}-\boldsymbol{K}_{G}^{\mathrm{g}-1,0}\left(\boldsymbol{K}_{G}^{0}\right)^{-1} \boldsymbol{F}_{G}^{0}
$$

\footnotetext{
${ }^{1}$ We underline, e.g., the vector of degrees of freedom $\underline{\boldsymbol{u}}_{G}^{0}$ to distinguish it from the three-dimensional displacement vector $\boldsymbol{u}_{G}^{0}=\boldsymbol{N} \underline{\boldsymbol{u}}_{G}^{0}$, where $\boldsymbol{N}$ is a matrix of GFEM shape functions.

[global-local'GFEM'duarte kim`R1 - August 18, 2007]
} 
The computation of $\widehat{\boldsymbol{K}}_{G}^{\text {g-l }}$ and $\widehat{\boldsymbol{F}}_{G}^{\text {g-l }}$ involves forward and backward substitutions using the factorization of $\boldsymbol{K}_{G}^{0}$, the stiffness matrix of the initial (coarse) global discretization. The system of equations (14) is very small since it involves only the enriched global-local degrees of freedom. The computation of $\underline{\tilde{u}}_{G}^{0}$ using (13) also involves forward and backward substitutions using the factorization of $\boldsymbol{K}_{G}^{0}$. Therefore, the computation of the solution of the enriched global problem, $\underline{\boldsymbol{u}}_{G}^{E}$, can be done very efficiently once the coarse global problem has been solved. ${ }^{2}$

\section{I.3. Numerical Integration of Global-Local GFEM Shape Functions}

In this section, we discuss how the proposed global-local enrichment functions can be integrated accurately and efficiently. The basic idea is to integrate on the global mesh using the local meshes. This is possible since these meshes are nested in the global mesh, as discussed above. Figure 13 illustrates the numerical integration scheme. Figure 13(a) shows a refined local problem where local solutions are available. These local solutions are used to enrich the nodes on the crack front which are represented by the square in Figure 13(b). The numerical integration of the global element enriched with the local solution is performed at the integration points of the small elements which are nested in the global element and originated from the local problem as shown in Figure 13(b). We call these small elements local problem descendants.

This procedure requires mappings from master coordinates in a local problem descendant to master coordinates in the global mesh. These coordinates are used in the computation of the global partition of unity $\varphi_{\alpha}$ in Equation (10). The mapping is implemented by first computing the global physical coordinates, $\boldsymbol{x}$, at an integration point in a local problem descendant, followed by the mapping of $\boldsymbol{x}$ to the master coordinates of an element from the global mesh. No search of global elements containing $\boldsymbol{x}$ is required thanks to the nesting of meshes as described above. The mapping to master coordinates can be computed in closed form for tetrahedral and triangular elements. The numerical experiments presented in Section 4.3 show that the overhead of these operations is small.

The integration order used on each local problem descendant nested in a global element is taken as the maximum of (i) the integration order of the global element disregarding its globallocal enrichment functions and (ii) the integration order of the local problem descendant plus one. The integration order from the local problem descendant is increased by one because the local solution is multiplied by a global partition of unity which is a linear polynomial. The above integration strategy provides exact integration of these GFEM shape functions.

Acknowledgments: The partial support of this work by the National Center for Supercomputing Applications and the University of Illinois at Urbana-Champaign, under the auspices of the NCSA/UIUC Faculty Fellows Program, and by the National Science Foundation under grant DMS-0611094 is gratefully acknowledged. The authors also wish to thank Prof. Ivo Babuška from the University of Texas at Austin for fruitful discussions during the course of this research.

${ }^{2}$ The approach described above is not used in our current implementation. The CPU time for the solution of the enriched global problem reported in Section 4 includes the factorization of entire global matrix defined in (12).

[global-local’GFEM'duarte'kim`R1 - August 18, 2007] 


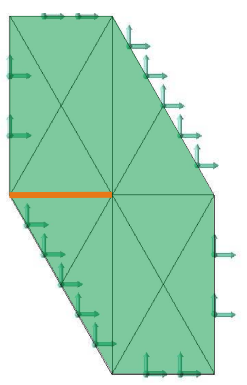

(a) Hp-refined local problem.

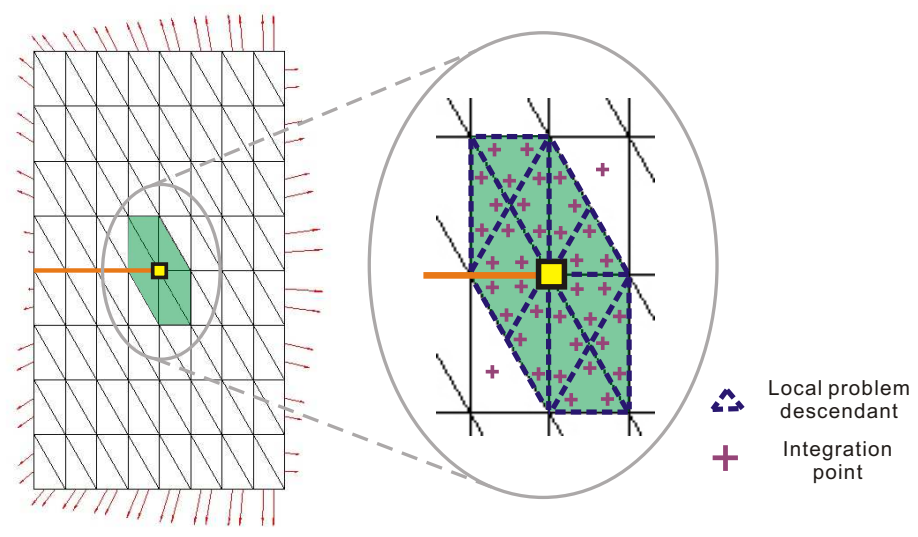

(b) Illustration of numerical integration scheme in the global elements enriched with local solutions.

Figure 13. Numerical integration procedure in the proposed $G F E M^{\mathrm{g}-1}$.

\section{REFERENCES}

[1] D. N. Arnold, A. Mukherjee, and L. Pouly. Locally adapted tetrahedral meshes using bisection. SIAM Journal of Scientific Computing, 22(2):431-448, 2000.

[2] I. Babuška. The finite element method with lagrange multipliers. Numer. Math., 20:179192, 1973.

[3] I. Babuška and B. Andersson. The splitting method as a tool for multiple damage analysis. SIAM journal on scientific computing, 26:1114-1145, 2005.

[4] I. Babuška, U. Banerjee, and J. E. Osborn. Survey of meshless and generalized finite element methods: A unified approach. Acta Numerica, 12:1-125, May 2003.

[5] I. Babuška, G. Caloz, and J. E. Osborn. Special finite element methods for a class of second order elliptic problems with rough coefficients. SIAM J. Numerical Analysis, 31(4):745-981, 1994.

[6] I. Babuška and J. M. Melenk. The partition of unity finite element method. Technical Report BN-1185, Inst. for Phys. Sc. and Tech., University of Maryland, June 1995.

[7] I. Babuška and J. M. Melenk. The partition of unity finite element method. International Journal for Numerical Methods in Engineering, 40:727-758, 1997.

[8] E. Bansch. Local mesh refinement in 2 and 3 dimensions. Impact of Computing in Science and Engineering, 3:181-191, 1991.

[9] R. Becker and P. Hansbo. A finite element method for domain decomposition with nonmatching grids. Technical Report RR-3613, INRIA, 1999. 
[10] T. Belytschko and T. Black. Elastic crack growth in finite elements with minimal remeshing. International Journal for Numerical Methods in Engineering, 45:601-620, 1999.

[11] C. Bernadi, Y. Maday, and A. Patera. A new non-conforming approach to domain decomposition: The mortar element method. In H. Brezis and J. L. Lions, editors, Nonlinear partial differential equations and their applications, pages 13-51. Pitman, 1994.

[12] G. F. Carey and J. T. Oden. Texas Finite Element Series Volume II-A Second Course. Prentice-Hall, New Jersey, 1983.

[13] E. G. D. Carmo and A. V. C. Duarte. A discontinuous finite element-base domain decomposition method. Computer Methods in Applied Mechanics and Engineering, 190:825-843, 2000.

[14] A.Th. Diamantoudis and G.N. Labeas. Stress intensity factors of semi-elliptical surface cracks in pressure vessels by global-local finite element methodology. Engineering Fracture Mechanics, 72:1299-1312, 2005.

[15] J. Dolbow, N. Moes, and T. Belytschko. Discontinuous enrichment in finite elements with a partition of unity method. Finite Elements in Analysis and Design, 36:235-260, 2000.

[16] C.A. Duarte. The hp Cloud Method. PhD dissertation, The University of Texas at Austin, December 1996. Austin, TX, USA.

[17] C.A. Duarte and I. Babuška. Mesh-independent directional p-enrichment using the generalized finite element method. International Journal for Numerical Methods in Engineering, 55(12):1477-1492, 2002.

[18] C.A. Duarte and I. Babuška. A global-local approach for the construction of enrichment functions for the generalized fem and its application to propagating three-dimensional cracks. In V.M.A. Leitão, C.J.S. Alves, and C.A. Duarte, editors, ECCOMAS Thematic Conference on Meshless Methods, Lisbon, Portugal, 11-14 July 2005. 8 pages.

[19] C.A. Duarte, I. Babuška, and J.T. Oden. Generalized finite element methods for three dimensional structural mechanics problems. In S.N. Atluri and P.E. O'Donoghue, editors, Modeling and Simulation Based Engineering, volume I, pages 53-58. Tech Science Press, October 1998. Proceedings of the International Conference on Computational Engineering Science, Atlanta, GA, October 5-9, 1998.

[20] C.A. Duarte, I. Babuška, and J.T. Oden. Generalized finite element methods for three dimensional structural mechanics problems. Computers and Structures, 77:215-232, 2000.

[21] C.A. Duarte, O.N. Hamzeh, T.J. Liszka, and W.W. Tworzydlo. A generalized finite element method for the simulation of three-dimensional dynamic crack propagation. Computer Methods in Applied Mechanics and Engineering, 190:2227-2262, 2001.

[22] C.A. Duarte, D.-J. Kim, and I. Babuška. Chapter: A global-local approach for the construction of enrichment functions for the generalized fem and its application to threedimensional cracks. In V.M.A. Leitão, C.J.S. Alves, and C.A. Duarte, editors, Advances 
in Meshfree Techniques, volume 5 of Computational Methods in Applied Sciences, The Netherlands, 2007. Springer. ISBN 978-1-4020-6094-6.

[23] C.A. Duarte, L.G. Reno, and A. Simone. A high-order generalized FEM for through-thethickness branched cracks. International Journal for Numerical Methods in Engineering, 2007. In Press (http://dx.doi.org/10.1002/nme.2012).

[24] C.A.M. Duarte and J.T. Oden. Hp clouds-a meshless method to solve boundary-value problems. Technical Report 95-05, TICAM, The University of Texas at Austin, May 1995.

[25] C.A.M. Duarte and J.T. Oden. An $h p$ adaptive method using clouds. Computer Methods in Applied Mechanics and Engineering, 139:237-262, 1996.

[26] C.A.M. Duarte and J.T. Oden. Hp clouds - an $h p$ meshless method. Numerical Methods for Partial Differential Equations, 12:673-705, 1996.

[27] J. Fish. The s-version of the finite element method. Computers and Structures, 43:539$547,1992$.

[28] B. Flemisch, M. A. Puso, and B. I. Wohlmuth. A new dual mortar method for curved interfaces: 2d elasticity. International Journal for Numerical Methods in Engineering, 63:813-832, 2005.

[29] P. Grisvard. Singularities in Boundary Value Problems. Research notes in Appl. Math. Spring-Verlag, New York, 1992.

[30] T. Y. Hou and X.-H. Wu. A multiscale finite element method for elliptic problems in composite materials and porous media. Journal of Computational Physics, 134:169-189, 1997.

[31] S.-H. Lee, J.-H. Song, Y.-C. Yoon, G. Zi, and T. Belytschko. Combined extended and superimposed finite element method for cracks. International Journal for Numerical Methods in Engineering, 59:1119-1136, 2004.

[32] J. M. Melenk and I. Babuška. The partition of unity finite element method: Basic theory and applications. Computer Methods in Applied Mechanics and Engineering, 139:289-314, 1996.

[33] N. Moes, M. Cloirec, P. Cartraud, and J.-F. Remacle. A computational approach to handle complex microstructure geometries. Computer Methods in Applied Mechanics and Engineering, 192:3163-3177, 2003.

[34] N. Moes, J. Dolbow, and T. Belytschko. A finite element method for crack growth without remeshing. International Journal for Numerical Methods in Engineering, 46:131-150, 1999.

[35] N. Moës, A. Gravouil, and T. Belytschko. Non-planar 3D crack growth by the extended finite element and level sets - Part I: Mechanical model. International Journal for Numerical Methods in Engineering, 53:2549-2568, 2002.

[global-local'GFEM'duarte`kim`R1 - August 18, 2007] 
[36] S. A. Nazarov and B. A. Plamenevsky. Elliptic Problems in Domains with Piecewise Smooth Boundaries, volume 13 of De Gruyter Expositions in Mathematics. Walter de Gruyter, Berlin, 1994.

[37] A. K. Noor. Global-local methodologies and their applications to nonlinear analysis. Finite Elements in Analysis and Design, 2:333-346, 1986.

[38] J. T. Oden and G. F. Carey. Texas Finite Element Series Volume IV-Mathematical Aspects. Prentice-Hall, New Jersey, 1983.

[39] J.T. Oden and C.A. Duarte. Chapter: Clouds, Cracks and FEM's. In B.D. Reddy, editor, Recent Developments in Computational and Applied Mechanics, pages 302-321, Barcelona, Spain, 1997. International Center for Numerical Methods in Engineering, CIMNE.

[40] J.T. Oden, C.A. Duarte, and O.C. Zienkiewicz. A new cloud-based $h p$ finite element method. Computer Methods in Applied Mechanics and Engineering, 153:117-126, 1998.

[41] J.T. Oden and C.A.M. Duarte. Chapter: Solution of singular problems using $h p$ clouds. In J.R. Whiteman, editor, The Mathematics of Finite Elements and Applications-Highlights 1996, pages 35-54, New York, NY, 1997. John Wiley \& Sons.

[42] C. Park, K, C. A. Felippa, and G. Rebel. A simple algorithm for localized construction of non-matching structural interfaces. International Journal for Numerical Methods in Engineering, 53:2117-2142, 2002.

[43] J. W. Park, J. W. Hwang, and Y. H. Kim. Efficient finite element analysis using mesh superposition technique. Finite Elements in Analysis and Design, 39:619-638, 2003.

[44] M. L. Parks, E. de Sturler, G. Mackey, D. D. Johnson, and S. Maiti. Recycling krylov subspaces for sequences of linear systems. Technical Report UIUCDCS-R-2004-2421, UILU-ENG-2004-1722, University of Illinois at Urbana-Champaign, Urbana, IL, March 2004.

[45] J.P. Pereira, X. Jiao, and C.A. Duarte. A robust geometry engine for modeling 3D crack problems with the generalized finite element method. In Seventh World Congress on Computational Mechanics, Los Angeles, CA, USA, 16-22 July 2006. Invited abstract.

[46] A. Quarteroni and A. Valli. Domain Decomposition Methods for Partial Differential Equations. Oxford University Press, 1999. ISBN: 0198501781.

[47] A. Simone, C.A. Duarte, and E. van der Giessen. A generalized finite element method for polycrystals with discontinuous grain boundaries. International Journal for Numerical Methods in Engineering, 67(8):1122-1145, 2006.

[48] B. Smith, P. Bjorstad, and W. Gropp. Domain Decomposition: Parallel Multilevel Methods for Elliptic Partial Differential Equations. Cambridge University Press, 2004. ISBN: 0521602866 .

[49] T. Strouboulis, I. Babuška, and K. Copps. The design and analysis of the generalized finite element mehtod. Computer Methods in Applied Mechanics and Engineering, 81(13):43-69, 2000.

[global-local`GFEM`duarte`kim`R1 - August 18, 2007] 
[50] T. Strouboulis, K. Copps, and I. Babuška. The generalized finite element method: An example of its implementation and illustration of its performance. International Journal for Numerical Methods in Engineering, 47(8):1401-1417, 2000.

[51] T. Strouboulis, K. Copps, and I. Babuška. The generalized finite element method. Computer Methods in Applied Mechanics and Engineering, 190:4081-4193, 2001.

[52] T. Strouboulis, L. Zhang, and I. Babuška. Generalized finite element method using mesh-based handbooks: Application to problems in domains with many voids. Computer Methods in Applied Mechanics and Engineering, 192:3109-3161, 2003.

[53] T. Strouboulis, L. Zhang, and I. Babuška. p-version of the generalized FEM using meshbased handbooks with applications to multiscale problems. International Journal for Numerical Methods in Engineering, 60:1639-1672, 2004.

[54] N. Sukumar, D. Chopp, N. Moes, and T. Belytschko. Modeling holes and inclusions by level sets in the extended finite element method. Computer Methods in Applied Mechanics and Engineering, 190:6183-6200, 2001.

[55] N. Sukumar, N. Moes, B. Moran, and T. Belytschko. Extended finite element method for three-dimensional crack modelling. International Journal for Numerical Methods in Engineering, 48(11):1549-1570, 2000.

[56] B. Szabo and I. Babuška. Finite Element Analysis. John Wiley and Sons, New York, 1991.

[57] B. I. Wohlmuth. A comparison of dual lagrange multiplier spaces for mortar finite element discretizations. Mathematical Modeling and Numerical Analysis, 36(6):995-1012, 2002.

[58] O. C. Zienkiewicz and R. L. Taylor. The Finite Element Method, $4^{\text {th }}$ Edition, volume I. McGraw-Hill, 1981. 